\title{
RECENT RESULTS FROM STUDIES OF ELECTRON BEAM PHENOMENA IN SPACE PLASMAS
}

\author{
TORSTEN NEUBERT and PETER M. BANKS \\ Space Physics Research Laboratory, Department of Oceanic, Atmospheric and Space Science, \\ University of Michigan, Ann Arbor, MI 48109-2143, U.S.A.
}

(Received 17 July 1991)

\begin{abstract}
Experiments involving the ejection of beams of electrons from spacecraft have been performed for more than 2 decades in order to study fundamental plasma physical processes as well as for a range of diagnostic- and application-oriented purposes. This paper reviews some of the key issues that have been pursued in the past 10 years. These inciude questions regarding spacecraft charging and beam dynamics, the interaction of beams with neutral gas and plasmas, and the electromagnetic radiation generated by continuous and pulsed electron beams. It is shown how our understanding of these phenomena has matured, thereby providing a solid foundation for future experiments involving the use of electron beams.
\end{abstract}

\section{INTRODUCTION}

The Universe as we know it is made up of neutral and ionized matter permeated by gravitational and electromagnetic fields. It is highly dynamic and in constant change as manifested by the birth and death of stars. One of the fundamental processes that arises naturally out of this dynamic interaction between matter and fields is the generation of electric currents. As charged particles tend to flow in force-free directions, currents tend to be magnetic field-aligned in which case they are often called "Birkeland" currents after Professor Birkeland who first suggested such currents flow in the Earth's magnetosphere (Egeland and Leer, 1986). Their existence is now firmly established and it has been found that they are intimately connected to "inverted V" events, the generation of aurora, and the generation of electromagnetic radiation in the range from a few Hertz to many megahertz. It is also thought that Birkeland currents are of importance throughout the Universe on a variety of scales up to galactic scales (Faelthammar, 1986; Peratt, 1986, 1988, 1990). It is therefore not surprising that the scientific community has shown considerable interest in performing space experiments which involve the ejection of electron beams and the formation of artificially generated currents. These socalled "active experiments" allow the study of fundamental plasma physical processes, thereby gaining quantitative information that is almost impossible to obtain when observing natural phenomena. With active experiments, the charged particle source (e.g. the electron beam) characteristics are known and provide a specific stimulus to the coupled response of electromagnetic fields and plasma. In many ways, active experiments provide an important calibration of natural phenomena.

There are a variety of additional objectives that also motivated past space experiments with charged particle beams. These include the probing of the Earth's magnetic field on a global scale by recording the echo of particles mirroring in the conjugate hemisphere as done in the $E C H O$ series of sounding rockets (Winckler et al., 1989 ; Hallinan et al., 1990), probing the local electric and magnetic field as done on GEOS 1 and 2 (Melzner et al., 1978 ; Junginger et al., 1984), and using pulsed electron beams as a means of generating electromagnetic radiation for purposes of communication between ionospheric spacecraft and receivers on conjugate spacecraft or on the Earth (e.g. Inan et al., 1984).

However, experience has shown that there are many technical problems associated with the successful ejection of charged particle beams in space and the study of these has taken considerable effort. A foremost challenge has been the charging of spacecraft platforms as a result of charged particle beam ejection. As pointed out by Linson (1982), an isolated spherical conductor of $1 \mathrm{~m}$ diameter will charge at a rate of $9 \mathrm{~V}$ $\mu \mathrm{s}^{-1}$ when a beam of $1 \mathrm{~mA}$ is ejected and no return currents from the ambient plasma are collected. Because of this rapid charging rate, and its influence on processes in the plasma surrounding the beam source, it is important to undertake experiments and theoretical studies aimed at understanding and mitigating the processes associated with electrical charging of the beam-emitting platform. In doing so the goal is both scientific and technical. On the one hand, to design successful space experiments with a charged particle beam apparatus it is important to understand 
the underlying physical processes linked with the beam emissions. On the other hand, it is also important to be able to achieve a high quality of the emitted charged particle beam for the purpose of probing nature with a beam of well-defined characteristics. The quest for such understanding involves an exciting body of basic physical processes, including the formation of large and complicated sheath regions of charged particle and electromagnetic field fluctuations, plasma-neutral gas interactions, waveparticle interactions within plasmas, and non-linear phenomena such as plasma wave turbulence, just to name a few.

In this paper it is our aim to review selected results of the 1980 s and to discuss future directions of active electron beam experiments in space. More information on beam experiments is found in the review paper by Winckler (1980), the monograph by Grandal (1982), the special issue of Advances in Space Research, edited by Brenning and Mendillo (1990), the special issue of Radio Science (1984), and the European Space Agency report by Burke (1983).

The organization of this paper is as follows: in Section 2 some basic processes associated with electron beam emissions are discussed. These are spacecraft charging, including the phenomenon of super charging and virtual cathode formation, beamatmosphere interaction (BAI), and beam/systemplasma interaction (BSPI). Then, in Section 3 we discuss the theory for electromagnetic radiation from continuous and pulsed electron beams and relate some experimental results obtained from space shuttle and sounding rocket experiments. Finally in Section 4 we discuss the current status of electron beam experiments and point out future directions for the field.

\section{BASIC PROCESSES}

When an electron beam is ejected from a spacecraft, the spacecraft potential will rise, drawing return current electrons from the ambient plasma. For low potentials, space charge in the plasma is small and the electrons collected by the spacecraft are those whose trajectories directly intersect the spacecraft structure. For potentials well above the ambient plasma thermal energy, an extended region of positive space charge will develop around the spacecraft. Ambient electrons that enter this sheath have a chance of reaching the payload through the combined action of the highly inhomogeneous electric field in the sheath and the ambient magnetic field. Thus, the effective current collecting area of the spacecraft is enhanced by the space charge sheath, thereby increasing the collected current.
The electron thermal current density $j_{o e}$ in an equilibrium plasma is given by :

$$
j_{\mathrm{oc}}=\mathrm{e} n\left\langle v_{e}\right\rangle / 4,
$$

where $e$ is the elementary charge, $n$ the plasma density, and $\left\langle v_{c}\right\rangle$ the average electron velocity. In a Maxwellian plasma the expression for $\left\langle v_{\mathrm{e}}\right\rangle$ is :

$$
\left\langle v_{\mathrm{e}}\right\rangle=\left(8 \mathrm{k} T / \pi m_{\mathrm{e}}\right)^{1 / 2},
$$

where $\mathrm{k} T$ is the electron energy and $m_{c}$ the electron mass. The ion thermal current density and ion velocity are given by similar expressions. While the temperature of the ionospheric plasma is fairly constant and approximately the same for ions and electrons to within a factor of $2-3$, the densities may vary many orders of magnitude. Typical values for the particle velocities and the peak densities encountered in the ionosphere and the corresponding current densities are given in Table 1.

The electron thermal current available to a sounding rocket payload at low potentials in the ionosphere is $2-10 \mathrm{~mA} \mathrm{~m}^{-2}$ depending on the plasma density and temperature. Beam experiments flown in the past have carried beam accelerators ejecting beams with currents up to several hundred milliamperes and individual electron energies up to $40 \mathrm{keV}$. Since current collecting areas on such platforms are seldom more than a few square meters, such payloads have often reached high positive potentials.

For most applications it is essential that the beam escapes the nearby region of the spacecraft in a wellknown and coherent manner, preferably with the full electron accelerator energy and small energy, spatial or temporal dispersion. In practice, observations and theoretical simulations indicate that beam emissions are accompanied by many dynamic charging processes, both of the platform, within the beam itself, and in the surrounding medium. Thus, platform charging and beam characteristics can have large dynamic fluctuations on time-scales of the order of plasma time periods (microseconds) and physical scales of the order of Debye lengths (millimeters). These micro-scale effects have the net consequence of d.c. charging of the spacecraft, reducing the beam escape current, degrading the average beam particle energy by an amount corresponding to the average spacecraft potential, and degrading the beam coherence.

A number of effects help to enhance the emitting platform return current above the limit set by ionospheric thermal current densities and thereby reduce platform charging. These effects are associated with interactions between the ambient plasma and any neutral gases surrounding the payload and the electron 
TABLE 1 .

\begin{tabular}{cccccc}
\hline $\mathrm{k} T(\mathrm{eV})$ & $n\left(\mathrm{~m}^{-3}\right)$ & $\left\langle v_{\mathrm{e}}\right\rangle\left(\mathrm{m} \mathrm{s}^{-1}\right)$ & $\left\langle v_{\mathrm{i}}\right\rangle\left(\mathrm{m} \mathrm{s}^{-1}\right)$ & $j_{\mathrm{oe}}\left(\mathrm{A} \mathrm{m}^{-2}\right)$ & $j_{\mathrm{oi}}\left(\mathrm{A} \mathrm{m}^{-2}\right)$ \\
\hline 0.1 & $5 \times 10^{11}$ & $2.1 \times 10^{5}$ & $1.2 \times 10^{3}$ & $4.2 \times 10^{-3}$ & $2.4 \times 10^{-3}$ \\
\hline
\end{tabular}

beam and charged platform. Specific effects include beam-atmosphere interactions (BAI) and beam-system plasma interactions (BSPI). The neutral gases involved may be the ambient atmosphere, outgassing from the platform, or attitude control thruster emissions. By various processes, these gases can be ionized and thereby create enhanced plasma densities in the vicinity of the payload. This component of the return current is sometimes called the "active" component to distinguish it from the "passive" component which is the current drawn to an anode immersed in a plasma. The process identified as the beam-plasma discharge (BPD), investigated in many chamber experiments (Bernstein et al., 1979), is thought to occur in space at relatively low altitudes (110-160 $\mathrm{km}$ ) (Linson, 1982) where the neutral gas density is relatively high. It will not be covered in this review.

\subsection{Passive current collection}

The problem of determining the current and potential existing between two concentric electrodes in an unmagnetized plasma was first discussed by Langmuir and Blodgett (1924). In their theory, an anode immersed in a plasma will develop a positive spacecharge sheath out to a distance $r_{s}$, the sheath edge, where the potential reaches the ambient plasma potential. At the sheath edge, thermal electron fluxes enter the sheath from the ambient undisturbed plasma, and the potential structure in the sheath as well as the location of the sheath edge are calculated by considering current continuity and Poisson's equation. The mixed boundary conditions are the thermal current density at the sheath edge and the potential of the conducting bodies. From this theory the current to a probe is space-charge limited. Langmuir and Blodgett (1924) gave the expression for the current to a probe at a positive potential $\omega_{0}$ in the case of spherical symmetry and no magnetic field :

$$
I_{\mathrm{scl}}=4 \pi \varepsilon_{0}\left(32 \mathrm{e} / 81 m_{c}\right)^{1 / 2} \Phi_{0}^{3 / 2} \alpha^{-2},
$$

where $\alpha$ is a geometrical parameter. It was assumed that the current was carried by electrons to an anode. If the probe sheath radius is much larger than the probe radius, $r_{p}$, the following expression was found for $\alpha$ :

$$
\alpha^{3 / 4}=1.11 r_{\mathrm{s}} / r_{\mathrm{p}}
$$

Since the current to the probe must equal the cur- rent across the probe sheath, we also have:

$$
I_{\mathrm{sil}}=4 \pi r_{s}^{2} j_{\mathrm{oe}}
$$

Combining equations (3)-(5) we find :

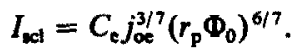

The expression for the ion current to a cathode is similar. For electrons the numerical value for $C_{e}$ is $6.946 \times 10^{-3}$, and for $\mathrm{O}^{+}$ions $C_{\mathrm{i}}$ is $3.674 \times 10^{-4}$, with the parameters of the equation given in SI units.

The current given in equation (6) has been found for an unmagnetized plasma. This condition can be regarded as a rather good approximation when considering ion collection by a negatively biased payload because of the large ion Larmor radius. For example, an $\mathrm{O}^{+}$ion which has just entered the sheath and obtained the energy of $1 \mathrm{eV}$ will already at that point have a Larmor radius of $13 \mathrm{~m}(B=0.44 \mathrm{G})$ which usually is large compared with the sheath dimension. The ions can therefore be regarded as unmagnetized in the sheath region. Electrons are, however, limited by the magnetic field in their motion perpendicular to the field, and thus the electron current that can be collected is reduced compared with the values predicted by equation (6).

Theories extending the work of Langmuir and Blodgett (1924) for the case of beam-enitting spacecraft in the ionosphere was put forward by Beard and Johnson (1961) (without magnetic field) and Parker and Murphy (1967) (with magnetic field). Parker and Murphy included the magnetic field effects by using the conservation of energy and angular momentum of particles collected by a sphere. The upper limit of electron current collection according to their results is :

$$
I / I_{0}<1+\left[8 \mathrm{e} \Phi_{0} /\left(m_{e} \omega_{c e}^{2} a^{2}\right)\right]^{1 / 2},
$$

where $I_{0}$ is the thermal electron current to the sphere at plasma potential, $\omega_{c e}$ is the electron gyrofrequency and $a$ is the radius of the sphere.

The space-charge limited current for an unmagnetized plasma given in equation (6) increases as $\Phi^{6 / 7}$ while the magnetic-field limited current in equation (7) increases $\Phi^{1 / 2}$. A consequence of this and of the differing dependence of system parameters appearing in the two expressions, the predicted current collection in the presence of a magnetic field is smaller than without a field as expected. 
Studies have been made in the past decade in space and in laboratory plasma chambers to test the validity of the estimates given in equations (6) and (7). For space experiments this task is not as simple as one would imagine because of the difficulty in measuring the spacecraft potential accurately during beam emissions. As we will show later, the ejection of charged particle beams has the effect of generating a turbulent region with enhanced energetic particle fluxes in the vicinity of the spacecraft. These fluxes contaminate the potential measurements, which are usually performed by measuring the potential difference between the rocket body and smaller spheres mounted on booms extending from the rocket. Furthermore, in many cases the sheaths are of such dimensions that the length of the floating probe booms is smaller than the sheath radius and thus the potential measurement does not reflect the complete potential difference between the ambient plasma and the spacecraft. Recently however, a technique has been developed which uses two payloads connected by a conducting, insulated wire. The payloads are allowed to drift apart up to many hundreds of meters and the potential of the beam-emitting payload is measured with respect to the remote tethered payload located outside of the disturbed region.

Such a technique was used in the CHARGE series of sounding rocket experiments (Sasaki et al., 1987, 1988; Myers et al., 1990). The sheath formation and the ion current collection to a negatively charged payload was studied by applying a potential difference of up to $460 \mathrm{~V}$ between the two tethered payloads, the mother payload biased negative and the daughter payload biased positive. The potential of each payload relative to the ambient plasma was estimated by noting that the total current to the structure must equal zero. As a consequence, the ion current to the negative mother balanced the electron current to the positive daughter. Since the thermal ion current density in the plasma is much smaller than the electron current density (Table 1) the mother charged to a large negative potential while the daughter charged to a small positive potential. In other words, by far the most of the $460 \mathrm{~V}$ potential drop was found in the positive ion sheath surrounding the mother payload.

The data obtained in the CHARGE-2 experiment was compared with computer calculations by the NASCAP/LEO code (Mandell et al., 1990; Neubert et al., 1990a). The code, in essence, simulated the space-charge limited current and the corresponding sheath structure as given by the theory of Langmuir and Blodgett (1924). However, the advantage of the code is that the actual shape of the spacecraft can be modelled rather than assuming an equivalent sphere or an infinite cylinder, as must be done when treating the problem analytically. Furthermore, the velocity of the spacecraft through the plasma could be included in the calculation. This is particularly important for ion-current collection since the spacecraft velocity is of the order of the ion thermal velocity and therefore significant wake and ram asymmetries are created in the ambient plasma density and the current collection. With the computer simulation it was also possible to estimate the effects of secondary electron emission caused by the bombardment of the rocket skin by incoming ions.

A contour plot of the sheath structure around the mother payload computed by the NASCAP/LEO code is shown in Fig. $1 a$ and $b$ for two values of the spacecraft potential: -30 and $-200 \mathrm{~V}$. The contour marked with the number 2 is the $-2 \mathrm{~V}$ contour, which is basically the sheath edge. The length of the rocket is $3.26 \mathrm{~m}$ and the diameter is $44 \mathrm{~cm}$. Four floating probes separated by $25 \mathrm{~cm}$ were mounted on a boom. The influence of these was also modelled by taking into account the internal resistance of the floating probe array. The probes can be seen at $z=9$ extending outward from the rocket.

The return current characteristic of the mother payload as a function of rocket potential was determined from the measurement of the current in the tether with gradually increased bias potentials applied between the mother and the daughter. The results are shown in Fig. 2 for two such experiments performed at 244 and $247 \mathrm{~km}$ altitude. Also shown are the results of the NASCAP/LEO code which show very good agreement with observations.

The study of current collection and sheath formation of payloads at negative potentials led to the following conclusions :

(1) Magnetic field effects on the ion motion in the sheath could be ignored, as expected. The sheath structure and the current collection was close to the predictions of the theory of space-charge limited flow with modifications listed below.

(2) The velocity of the rocket had the effect of increasing the ion-current collection by as much as $40 \%$ at low potentials, decreasing to $15 \%$ at higher potentials (Mandell et al., 1990). At the time of the experiments the rocket velocity was $580 \mathrm{~m} \mathrm{~s}^{-1}$.

(3) Laboratory experiments on secondary electron yields arising from alkali metal ions impacting on aluminum oxidized surfaces show that significant numbers of secondary electrons are emitted for potentials above a threshold of $200 \mathrm{~V}$ and that the yield reaches 0.5 electrons per impacting ion at $400 \mathrm{~V}$ (Dietz and Sheffield, 1975). To the best of our knowledge, 
(a)

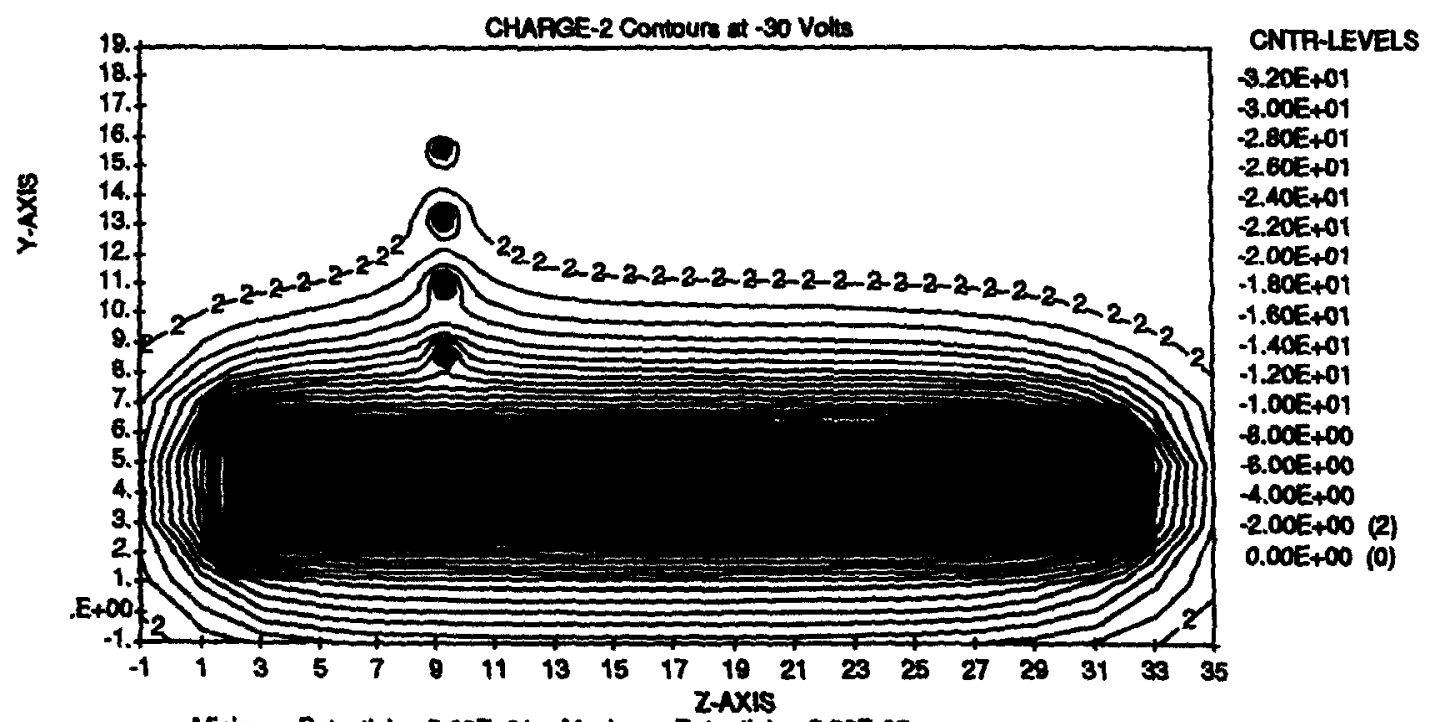

Minimum Potential $=3.005+01$ Maximum Potenild $=5.305-05$

$-1.00<Z<35.00,-1.00<Y<19.00$, CUTPLNE OFFSET $X=0.00 \quad 817911350 \times 5$

(b)

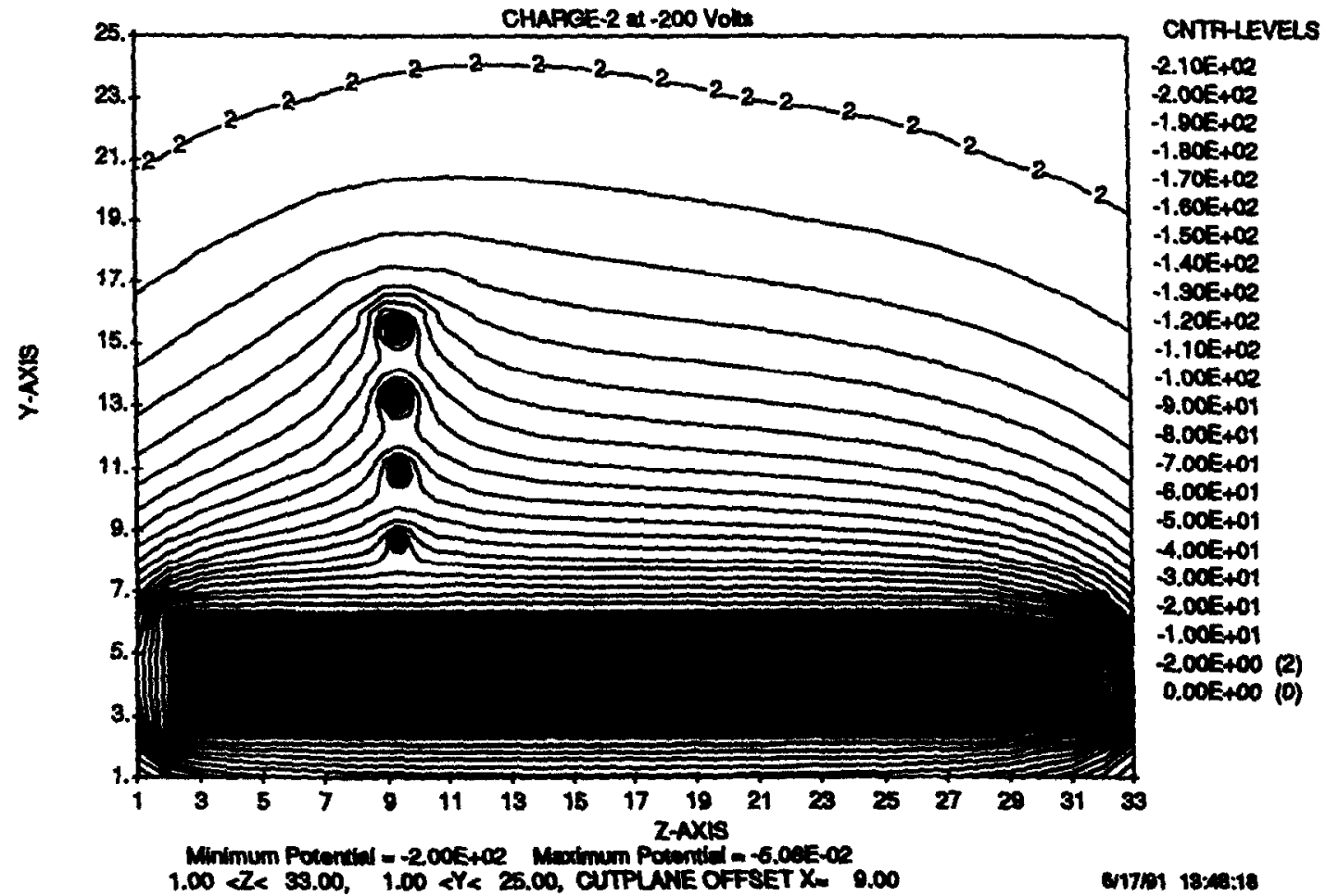

Fig. 1. NASCAP/LEO SMULATION OF THE POTENTIAL STRUCTURE AROUND THE CHARGE-2 MOTHER PAYLOAD WHEN BLASED TO (a) $-30 \mathrm{~V}$ AND (b) $-200 \mathrm{~V}$.

The $x$ and $y$ axes are in units of $10.8 \mathrm{~cm}$. The $-2 \mathrm{~V}$ potential contour is marked with the number 2 (from Neubert et al., 1990a). 


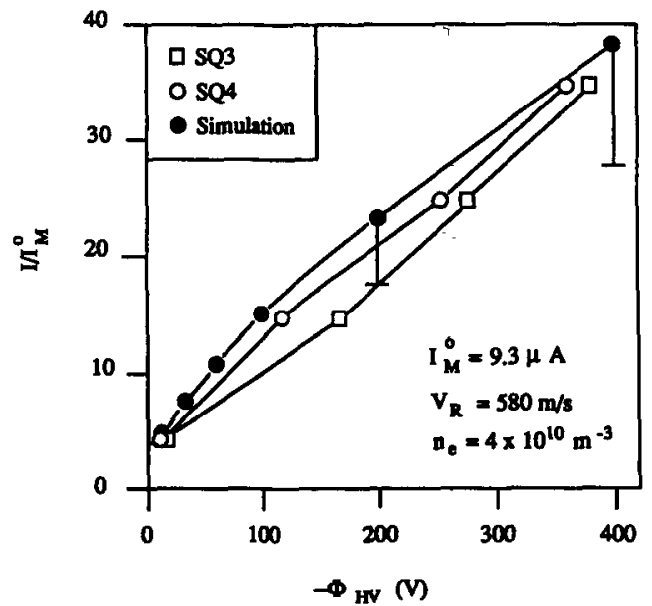

Fig. 2. THE ELECTRON RETURN CURRENT-PAYLOAD POTENTIAL CHARACTERISTIC OF THE CHARGE-2 MOT́TER PAYLOAD (FROM NEUBERT et al., 1990a).

no studies have been performed for $\mathrm{O}^{+}$ion beams bombarding oxidized metal surfaces. However, reports of other combinations of ion beams and surfaces indicate that the values given above are applicable to oxidized metal surfaces (Szapiro et al., 1988; Szapiro and Rocca, 1989; Utterback and Miller, 1961 ; Hayden and Utterback, 1964 ; Amme, 1969). A review is given in Langley et al. (1984). The NASCAP/ LEO simulations and comparison with experimental data indicated that secondary electrons were emitted during the negative bias operations of $C H A R G E-2$ roughly as predicted by laboratory data.

An electron beam source mounted on the CHARGE-2 mother payload emitted $1 \mathrm{keV}$ electrons with currents up to $46 \mathrm{~mA}$ as measured by on-board diagnostics. The current-voltage characteristic of the daughter payload (electron collection at positive potential) was obtained for beam-emission sequences executed with low resistance in the tether circuit. Comparison of the observations and the theoretical estimates given by equations (6) and (7) was reported by Myers et al. (1989). The key results are shown in Fig. 3. The model estimates were found by approximating the short cylindrical daughter payload $(44 \mathrm{~cm}$ diameter, $82 \mathrm{~cm}$ long) with an equivalent (equal area) sphere. As can be seen from the figure, the observed electron return current and the Parker and Murphy model estimates are in close agreement and substantially smaller than the Langmuir-Blodgett predictions.

With the assumption of current continuity (ejected current equals collected current) and beam escape, the

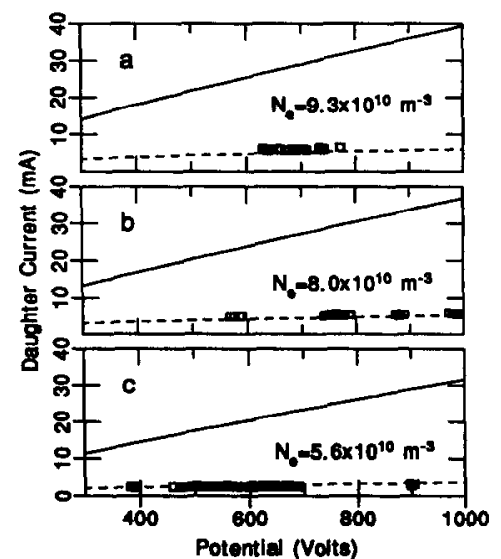

Fig. 3. CURRENT COLLECTION BY THE CHARGE-2 DAUGHTER PAYLOAD VS VEHICLE POTENTIAL COMPARED WITH : THE LANGMUIR AND BLODGETT MODEL (SOLID LINE) AND THE PARKER AND MURPHY MODEL (DASHED LINE) (FROM MYERS et al., 1989).

return current-voltage characteristic of the mother payload could also be determined. The observations were compared with the models of equations (6) and (7), again approximating the mother payload as an equivalent sphere. It was found that at high altitudes (above $240 \mathrm{~km}$ ) the electron return current was again well below the estimate of Langmuir and Blodgett, but above the upper limit of Parker and Murphy (Myers et al., 1989, 1990; Mandell et al., 1990). At low altitudes, however, the return current increased above even the Langmuir and Blodgett model estimate. The reason for this is the increased influence of the ambient neutral atmosphere and the BAI process, to which we will return in the following subsection.

Mandell et al. (1990) studied the return current collection of the CHARGE-2 mother payload at high altitudes using the true shape of this body. These computer estimates were in better agreement with the observations than estimates based on the Parker and Murphy model. Mandell $e t$ al. concluded that the difference arose from the fact that the Parker and Murphy model assumes a spherical body. Thus, although the daughter payload was a short cylinder, it was well approximated by a sphere. The mother payload, in contrast, was a long cylinder which was oriented perpendicular to the ambient magnetic field and this significantly increased the return current over the Parker and Murphy model values.

Study of current collection during application of passive positive potentials led to the following conclusions :

(4) For spherical payloads, the electron return cur- 
rent is magnetically limited and close to the Parker and Murphy limit even during simultaneous electron beam emissions, provided the experimental altitude is above $240 \mathrm{~km}$. (Rocket apogee was at $260 \mathrm{~km}$ so no information pertaining to the topside ionosphere was obtained.)

(5) A non-spherical payload can collect electron return currents somewhat in excess of the Parker and Murphy limit, as estimated by approximating the payload with an equivalent area sphere. The difference can be attributed to the fact that the Parker and Murphy model relies on the assumption of symmetry in the ambient magnetic field.

Laboratory and rocket experiments on charging phenomena performed in Japan have been reviewed by Kawashima (1982). The observations presented there are generally in accord with the conclusions reached above, provided the system is in a regime where surrounding neutral gases are not ionized. As will be discussed in the following sections, neutral gases may, in fact, play a very important role in space-borne electron beam experiments.

\subsection{Beam-atmosphere interactions}

The beam-atmosphere interaction (BAI) involves the collisional ionization of the ambient atmosphere by energetic electrons. It is a process which occurs naturally on auroral field lines where electron beams precipitate into the upper atmosphere generating aurora and enhancing the ambient plasma densities. The excitation of aurora is one of the natural phenomena that can be studied with the use of artificial electron beams, as will be done in the $A T L A S-1$ space shuttle mission scheduled for early 1992 (Taylor et al., 1991).

For the purpose of studying the effect of BAI on charging levels and return current collection of space craft, the CHARGE-2 sounding rocket experiment was the first to allow separate measurement of the active and the passive component of the return current (Neubert et al., 1990b). The experimental configuration is shown in Fig. 4, which illustrates the two payloads and the current system during electron beam ejections from the mother. From the measurements of the beam current, $I_{\text {beam }}$, and the tether current, $I_{\text {tether }}$, the return currents to the payloads can be determined by assuming that the beam current escapes the nearenvironment of the mother:

$$
\begin{gathered}
I_{\mathrm{D}}=I_{\text {tether }} \\
I_{\mathrm{M}}=I_{\text {beam }}-I_{\text {tether }},
\end{gathered}
$$

where $I_{\mathrm{D}}$ is the return current to the daughter and $I_{M}$ the current to the mother. The daughter current represents the passive current component since the
Chare 2

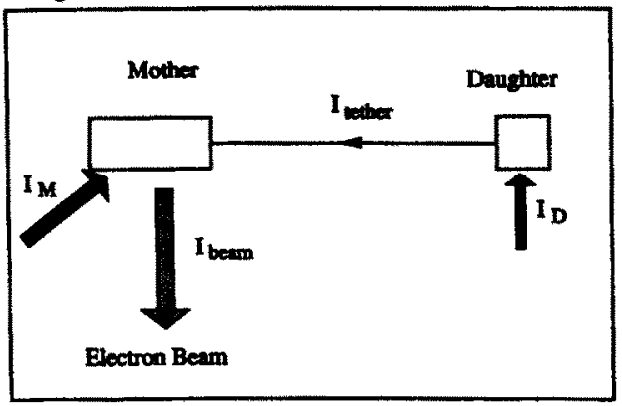

FIG. 4. CONFIGURATION OF THE CHARGE-2 PAYLOAD AND THE AVERAGE ELECTRON CURRENT SYSTEM AROUND THE PAYLOAD DURING AN ELECTRON BEAM EXPERIMENT (FROM NEUBERT et al., 1990b).

daughter is located far from the mother and outside the disturbed region surrounding the mother and the electron beam, while the current to the mother represents the sum of the passive and active components. Since the passive component can be deduced from the daughter measurement, the active component can be extracted from the mother return current.

The observations of current collection during the CHARGE-2 flight are summarized in Fig. 5. This shows the fraction of the beam current collected by the daughter as function of altitude. The labels SQ2SQ6 mark the beam-emission sequences performed

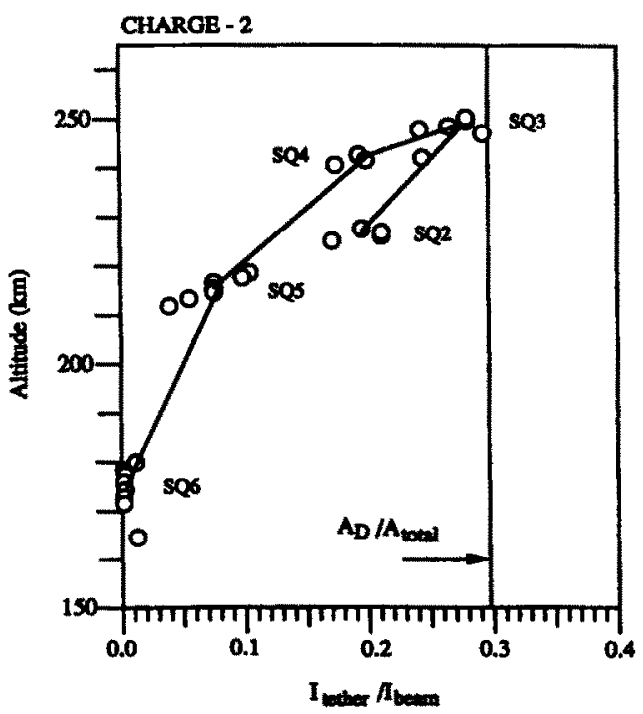

FIG. 5. CHARGE-2 OASERVATIONS OF THE TETHER TO BEAM CURRENT RATIO AS A FUNCTION OF ALTITUDE.

Also indicated is the ratio of the daughter area to the total area of the two payloads (from Neubert et al., 1990b). 
during the flight numbered in time sequence. Also indicated is the fixed ratio of daughter current collection area to the total collecting area of the two payloads. At high altitudes, the fraction of the return current collected by the daughter is close to the ratio of the two areas. This is expected for passive current collection. At lower altitudes, a progressively smaller fraction of the total return current is collected by the daughter. Assuming that the beam escapes, this implies that progressively more current must be collected directly by the mother. This can only happen if the active component of electron current increases.

Since the active current component increases with decreasing altitude it was suggested by Neubert $e t$ al. (1990b) that the active component was due to ionization created in a BAI process. In order to quantify the increased current due to this process, a computer code, first developed for the study of the generation and transport of photoelectrons in the upper atmosphere (Banks and Nagy, 1970) and later for auroral electron fluxes (Banks et al., 1974), was modified by Neubert $e t$ al. for the purpose of modelling the behavior of magnetic field-dominated electrons ejected from a spacecraft and scattered back and forth along magnetic field lines by collisions with atmospheric gases. Primary and secondary electrons scattered back to the payload would constitute the active source of return electron current.

The code solves two first-order, non-linear differential equations in the differential energy flux streaming along an ambient magnetic field. The equations coupling the forward flux, $\Phi^{+}$, with the backward flux, $\Phi^{-}$, are described by :

$$
\begin{gathered}
\Phi^{+\prime}(z, E)=-\sigma_{2}(z, E) \Phi^{+}(z, E)+\sigma_{1}(z, E) \Phi^{-}(z, E) \\
+Q^{+}(z, E)-L(E) \Phi^{+}(z, E) \\
\Phi^{-\prime}(z, E)=\sigma_{2}(z, E) \Phi^{-}(z, E)-\sigma_{1}(z, E) \Phi^{+}(z, E) \\
-Q^{-}(z, E)+L(E) \Phi^{-}(z, E) .
\end{gathered}
$$

Here $\Phi^{\prime}$ denotes the derivative of $\Phi$ with respect to $z$, which is the dimension in the direction of the magnetic field. $\sigma_{2}$ is the cross-section describing the loss in the electron energy range $E$ to $E+\mathrm{d} E, \sigma_{1}$ is the crosssection describing the elastic backscattering of electrons, $Q$ is the electron production rate due to ionization and cascading of electrons down in energy, and $L$ describes the loss of flux out of the beam fiuxtube induced by the motion of the spacecraft perpendicular to the magnetic field.

The problem is treated in one dimension and is applied to the situation of electrons emitted from a spacecraft by modelling the fluxes that are confined to the magnetic flux-tube encompassing the beam. It is assumed that the beam and the secondary electrons fill a flux-tube with a certain cross-sectional dimension and that the fluxes are homogeneous within this fluxtube. From experiments, an estimate of the width of the beam flux-tube is 4 beam electron Larmor radii at $90^{\circ}$ pitch angle (Frank et al., 1989; Winckler et al., 1989). The loss term $L$ describes the loss of flux out of the sides of the flux-tube caused by the motion of the spacecraft perpendicular to the magnetic field.

To model typical conditions, equations (10) and (11) were solved numerically for a $1 \mathrm{keV}$ electron beam of $100 \mathrm{~mA}$ current emitted downwards from an altitude of $260 \mathrm{~km}$ over White Sands, NM, at midnight. The MSIS/86 model (Hedin, 1987) was used for the neutral atmosphere $\left(\mathrm{N}_{2}, \mathrm{O}_{2}, \mathrm{O}\right)$ and the IRI model (Bilitza, 1986) was used for the ionosphere. The IRI model is needed to model Coulomb collisions.

The differential electron energy flux at the spacecraft altitude is shown in Fig. 6. The upward flux is the flux returning to the spacecraft from below. A portion of this flux will in general be collected by the spacecraft and form the active component of the return current, while the rest will reach locations above the spacecraft and interact with the neutral constituents here. In Figs 6 and 7 it is assumed that the portion of the flux collected by the spacecraft can be ignored relative to the total flux.

The downward flux consist of two contributions.

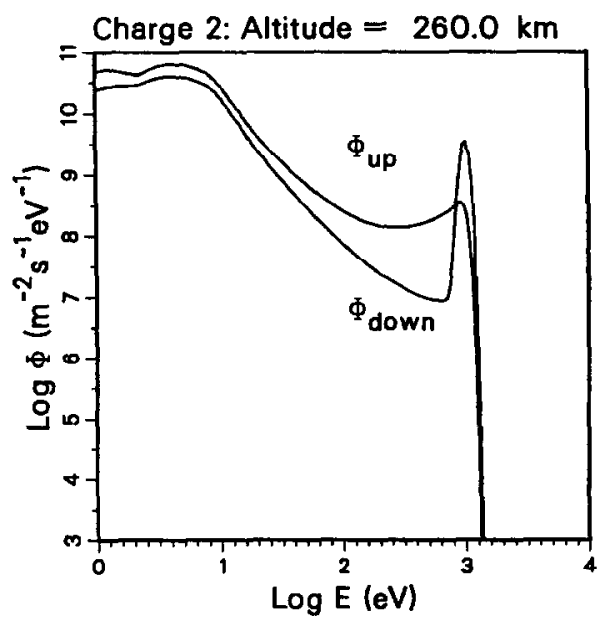

FIG. 6. MOdEL ESTIMATES OF THE UPWARD AND DOWNWARD DIFFERENTIAL ELECTRON FLUX AS A FUNCTION OF ENERGY AT THE SPACECRAFT ALTITUDE.

The beam is emitted downward parallel to the magnetic field with an energy of $1 \mathrm{keV}$ and a current of $100 \mathrm{~mA}$ (the fluxes scale linearly with beam current). The primary beam is seen as the peak at $1 \mathrm{keV}$ in the downward flux (from Neubert $e t$ al., 1990b). 
One is the electron beam flux, which is modelled as Gaussian in energy around the $1 \mathrm{keV}$ beam energy, and the other is the secondary electrons generated at lower energies and propagating in the same direction as the beam electrons from locations above the spacecraft. Thus, one will in general expect a hot component of electron fluxes to impinge on the spacecraft from both hemispheres. This is in accordance with observations made in the ECHO-2 experiment (Winckler et al., 1975).

Figure 7 shows the fluxes as a function of altitude for $1 \mathrm{keV}$ and $100 \mathrm{eV}$ electrons. As can be explained by electron free-path considerations, energetic electron

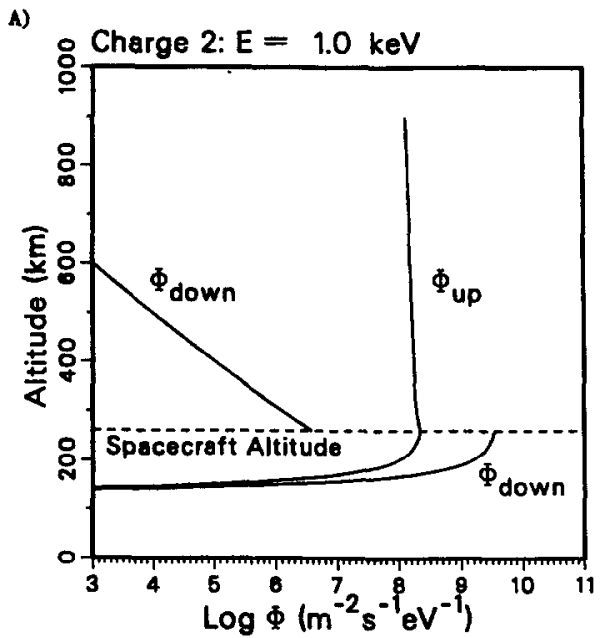

B)

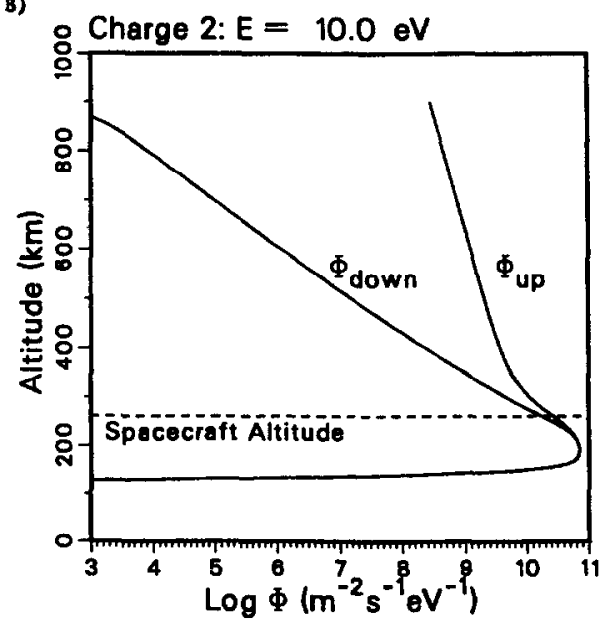

FIg. 7. MODEL ESTIMATES OF THE DIFFERENTIAL ELECTRON FLUXES AS A FUNCTION OF ALTITUDE FOR THE SAME PARAMETERS USED IN FIG. 6.

(a) 1-keV electrons and (b) $10-\mathrm{eV}$ electrons (from Neubert et al., 1990b). fluxes are generated along the magnetic field to considerable distances from the spacecraft. This is in accordance with optical observations in other experiments which found streaks of optical emissions aligned with the magnetic field and extending far from the payload and its sheath (O'Neil et al., 1978a,b; Winckler et al., 1989).

At low altitudes the flux becomes omnidirectional and approaches zero because of the progressively shorter mean free path in the lower thermosphere. Above the spacecraft, the upward flux decreases relatively slowly with altitude because of the increased mean free path which allows the electrons to escape without significant collisions. Similarly, the downward flux from above the spacecraft increases from zero at high altitudes (a boundary condition) to large values at low altitudes.

The active return current estimated to reach the CHARGE-2 rocket mother payload from such model calculations has been found to be consistent with the observed magnitude of the active current. Thus, there was no need to involve other processes for this experiment. However, at higher altitudes and for spacecraft velocities across the magnetic field typical of orbiting platforms, the active return current component due to BAI becomes small. In order to estimate the BAI currents to other types of ionospheric spacecraft a series of calculations were performed for a range of altitudes, spacecraft sizes, and velocities (Neubert and Banks, 1990). Noting that the BAI return current is directly proportional to the emitted beam current, the BAI effect can be quantified by a gain factor $\Lambda$ which is the ratio of the return current to the ejected beam current at the spacecraft altitude. The loss to the fluxtube incurred by the motion of the spacecraft contained in the term $L$ of equations (10) and (11) can be described by the parameter $v$ :

$$
v=v_{\perp} / \mathrm{d} y,
$$

where $v_{\perp}$ is the cross-field velocity of the spacecraft and $\mathrm{d} y$ is the cross-field dimension of the beam disturbance in the direction of motion.

A summary of the BAI study is shown in Fig. 8 where the gain factor $\Lambda$ is plotted as function of $v$ for three different altitudes. The gain factor is split into two parts describing the fluxes from above and below the spacecraft. As expected, when $v$ becomes large, $\Lambda$ decreases. Similarly, when the altitude increases, $\Lambda$ decreases. At the bottom of the figure is indicated the range in $v$ that can potentially be covered by various ionospheric spacecraft. An array of 10 electron guns along the space station may, when the long axis of the station is aligned with the velocity vector, result in a large value of $\mathrm{d} y$, say $100 \mathrm{~m}$. The ionospheric orbital 


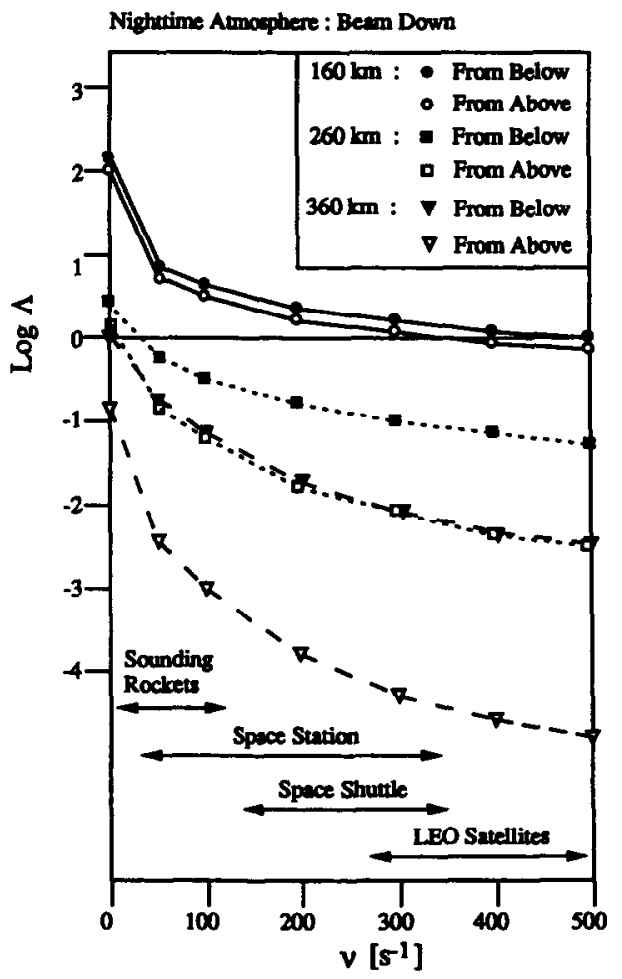

Fig. 8. MOdEL ESTIMATES OF THE BEAM ATMOSPHERE INTERACTION (BAI) GAIN FACTOR $\Lambda$ AS A FUNCTION OF THE SPACECRAFT PARAMETER $v$ (FROM NEUBERT AND BANKS, 1990).

velocity is typically $7 \mathrm{~km} \mathrm{~s}^{-1}$ with perpendicular velocities down to $3.5 \mathrm{~km} \mathrm{~s}^{-1}$. The minimum $v$ in this case is $35 \mathrm{~s}^{-1}$.

Since the spacecraft potential sheath is generally smaller than the beam flux-tube dimension, $\Lambda$ must be somewhat larger than 1 in order for the BAI return current to balance the beam current. From Fig. 8 we estimate that at $200 \mathrm{~km}$ altitude, a spacecraft with $v$ of $50 \mathrm{~s}^{-1}$ will just be able to collect the complete return current from the BAI.

It is also the case that an energetic electron wake exists behind an orbiting or otherwise moving payload emitting a steady electron beam. This wake is aligned along the magnetic flux-tubes traversed by the beam. The width of the region is of the order of four gyroradii, based on measurements by Frank et al. (1989) and Winckler et al. (1989). The length of the wake behind the payload depends on the time taken for the atmospherically scattered electrons to lose their excess kinetic energy or to escape from the atmosphere. The energy degradation time, $T$, depends on the magnitude of the inelastic cross-section, $\sigma_{1}$, the neutral gas density, $n$, and the electron velocity, $v_{e}$, according to the relation $T \approx 5 / n \sigma_{1} v_{\mathrm{e}}$, assuming that about five collisions are needed to bring an average energetic electron down to thermal electron energies. For conditions at $220 \mathrm{~km}$, for example, $T \approx 0.03 \mathrm{~s}$. For an orbiting payload at this altitude in the ionosphere, the energetic electron wake zone would extend approximately $1.2 \mathrm{~km}$ behind the vehicle. At higher altitudes, the wake length is determined by the speed at which electrons leave the atmosphere. In this case elastic scattering dominates, but numerical models are necessary to determine counteracting effects of the atmospheric albedo for downward moving electrons and net escape from the atmosphere.

The plasma density enhancement and optical emissions from the BAI are phenomena which will be explored in the $A T L A S$-1 space shuttle mission (1992) and the CHARGE-2b sounding rocket experiment planned for the spring of 1992. The experiments can study auroral processes in a quantitative manner and can in addition give new information on the physics of plasma density structures in the lower ionosphere and their influence on radio wave propagation (Banks and Gilchrist, 1985). Using the model described by equations (10) and (11), both plasma density enhancements along the beam flux-tube and optical emission rates can be calculated. Examples of the plasma density enhancement created by electron beam ejection from $200 \mathrm{~km}$ altitude are shown in Fig. 9 for two electron beam energies. The time constant for recombination is inversely proportional to the electron density and is about $7.7 \mathrm{~s}$ for $10^{6} \mathrm{~cm}^{-3}$ increasing to about 1 min when the density has decayed to $10^{5} \mathrm{~cm}^{-3}$. At this point the density is still a factor 100 larger than the background density.

In situ observation of plasma density enhancements is difficult. However, observing the phase shift of the telemetry signal between a mother and daughter pair, where one of the payloads carries an electron accelerator, estimates of the local plasma density enhancement can be made as was done in the MAIMIK experiment (Friedrich et al., 1991). The upper estimates found here are consistent with the estimates shown in Fig. 9.

The results from the BAI studies were:

(6) The interaction of artificial electron beams with the neutral atmosphere can be modelled, thereby giving quantitative estimates of enhanced return currents, as well as plasma density enhancements, optical emissions, electron differential energy fluxes, etc. along the magnetic flux-tubes containing the source electrons.

\subsection{Beam/system-plasma interactions}

The ejection of an electron beam from a spacecraft strongly perturbs the local plasma environment. In 

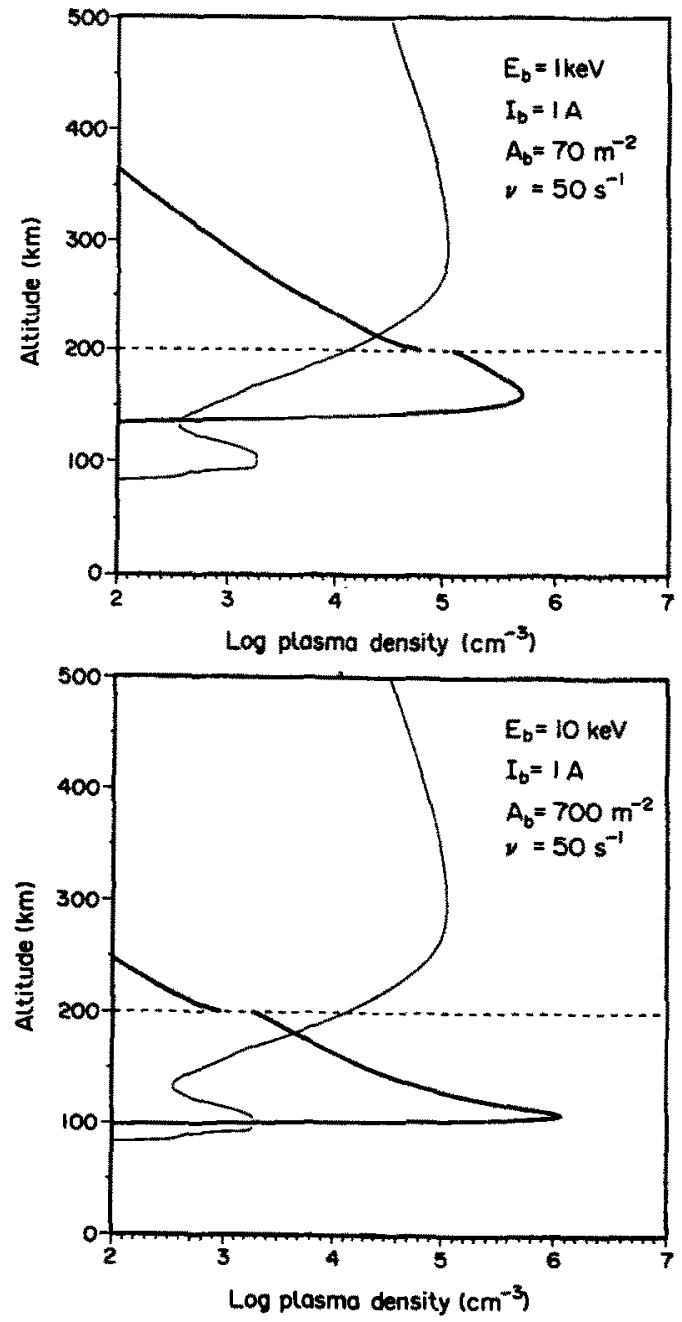

Fig. 9. Model estimates of THE PLASMa DENSITY IN THE BEAM FLUX-TUBE AS A FUNCTION OP ALTITUDE (HEAVY LINE) AND AMRIENT PLASMA DENSITY (THIN LINE).

The spacecraft altitude is indicated by the dashed line (from Neubert and Banks, 1990).

addition to the ionization of the ambient neutral atmosphere mentioned above, beam ejection results in plasma wave generation and plasma heating in a region surrounding the spacecraft and the beam fluxtube (Cartwright et al., 1978; Arnoldy and Winckler, 1981 ; Arnoldy et al., 1985). Many of the processes involved in this interaction are also found in conjunction with auroral precipitation of energetic electrons (Gurnett et al., 1986; Frank et al., 1989).

In the past, these processes have been studied in the laboratory as well as in space. The advantage of space experiments is the absence of walls and their con- taminating effects, which allows the study of largerscale phenomena such as the electromagnetic radiation from electron beams which usually have very long wavelengths compared with other scales in a plasma (Debye length, Larmor radius, etc.). On the other hand, a disadvantage is that it is more difficult to get detailed measurements. In the laboratory, the experiments can be repeated many times and measurements can be performed at different locations giving good temporal and spatial coverage. In space, this problem has been approached with the use of two or more separate payloads, one carrying a source and all payloads carrying a selection of plasma diagnostic instrumentation. Such configuration has proved very useful as demonstrated by the recent $E C H O-6$ and -7 sounding rocket experiments (Winckler et al., 1989; Erickson and Winckler, 1990) and the Spacelab-2 flight of the space shuttle which released a plasma diagnostics package to free-fly during electron beam emission from the space shuttle orbiter (Gurnett et al., 1986; Reeves et al., 1988a).

The plasma disturbances are a consequence of the sudden ejection of large amounts of charge which create charge imbalances and large transient electric fields at beam turn on and off. In addition, the drift relative to the ambient plasma of the energetic primary beam electrons and the lower energy return current electrons and secondary electrons generate plasma waves in a broad frequency range. The hot plasma region around the beam flux-tube was investigated in the $E C H O-7$ experiment. Here three sub-payloads were injected upwards relative to the main payload at various angles to the magnetic field. The main payload carried an electron beam accelerator ejecting beams at a range of energies and currents (up to $36 \mathrm{keV}$, $180 \mathrm{~mA}$ ) as well as beam pitch angles. The turbulent region as observed from one of the sub-payloads, the Plasma Diagnostics Package (PDP), is shown in Fig. 10 (Winckler et al., 1989). In the figure, the floating potential of the $P D P$ is shown as function of perpendicular distance to the beam flux-tube for a range of beam pitch angles (the floating potential is proportional to the temperature of the plasma). The dimension of the disturbed region generally increases with beam pitch angle. It is largest for $75^{\circ}-85^{\circ}$ pitch angle even though the beam in this case ejected downwards and away from the $P D P$. This indicates that much of the disturbance is accounted for by secondary and scattered electrons in the flux-tube rather than the primary beam electrons.

In the disturbed region around the beam flux-tube, low frequency electrostatic waves are excited in the range from essentially zero frequency and up to harmonics of the electron gyrofrequency (Shawhan et al., 


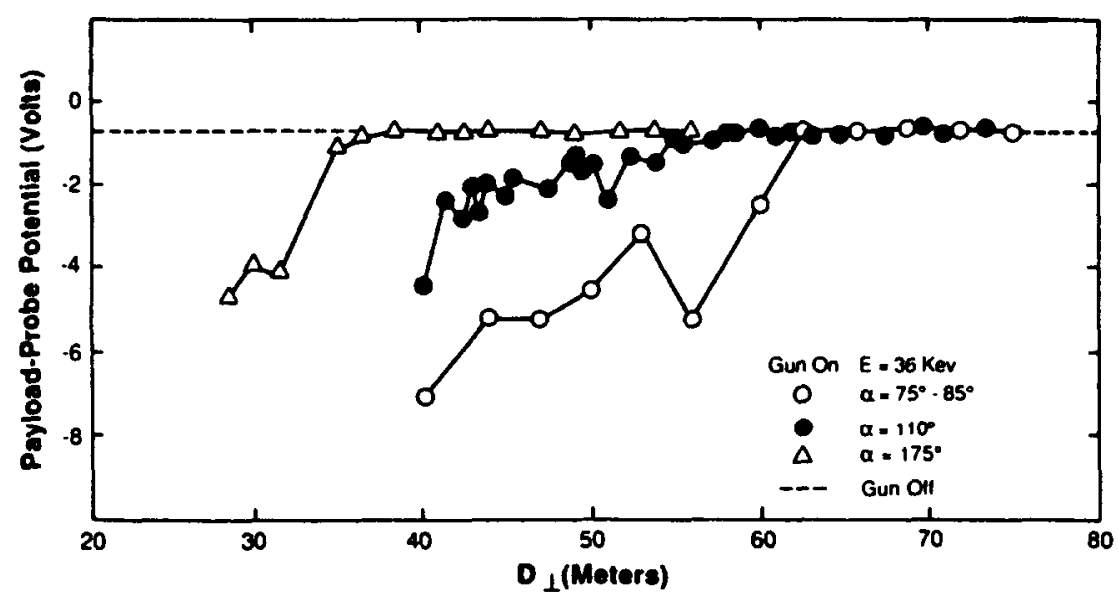

FIG. 10. ECHO-7 OBSERVATIONS OF THE HOT PLASMA FOR THREE INJECTION PITCH ANGLES IN TERMS OF THE FLOATING POTENTIAL DECREASE OF THE PDP PAYLOAD bOdY, PROPORTIONAL to $k T$, as a FUNCTION OF DISTANCE TRANSVERSE FROM THE BEAM INJECTION FIELD LINE, ALL, FOR 36-kV, $180 \mathrm{~mA}$ INJECTIONS (FROM WINCKLER et al., 1989).

1984; Neubert et al., 1986, 1991 ; Abe et al., 1988; Frank et al., 1989). The low frequency modes have been studied in the laboratory (Gekelman and Stenzel, 1978; Stenzel, 1978a,b) and in computer simulations (Okuda and Ashour-Abdalla, 1988). The concensus is that the broad-banded noise below the lower hybrid frequency is ion acoustic noise generated by lower energy electrons flowing primarily as return currents to the payload. The disturbed region is, however, not symmetric around the magnetic field. Due to the motion of a spacecraft perpendicular to the field, there exists a turbulent wake region behind the beam (Frank et al., 1989; Reeves et al., 1990a). In the beam region, as in the wake, elevated electron temperatures and low frequency wave turbulence are found. The low frequency waves are then generated not just by return current electrons but, more generally, by lower energy electrons.

Electromagnetic broad-banded noise in the whistler mode extending from the lower hybrid frequency and to the electron gyrofrequency is thought to be generated by the primary beam electrons (Gurnett $e t$ al., 1986). We return to this point in the following section.

Localized primarily to the turbulent beam region are waves around the electron gyrofrequency and its harmonics, the plasma frequency and the upper hybrid frequency (Cartwright and Kellogg, 1974; Gurnett et al., 1986; Neubert et al., 1991). Computer simulations have shown that these high frequency wave modes are generated by the primary beam electron motion as well as by the induced azimuthal $E \times B$ drift of beam electrons and ambient plasma electrons around the beam. The electric field here is either the radial field caused by the excess negative charge in the beam column or the electric field from waves in resonance with the particles (Winglee and Kellogg, 1990 ; Okuda and Ashour-Abdalla, 1990). As a result, the beam electrons experience energy loss and scattering.

Computer particle simulations have proven to be very useful in studying spacecraft charging, return current collection, and beam coherence. Using 2-D simulations (three velocity components) Winglee and Pritchett (1988) studied many aspects of the dynamics of electron beams emitted from spacecraft. In Fig. 11 is shown the response of the plasma to the ejection of an electron beam at a time $\omega_{\mathrm{pb}} t=120$, where $\omega_{\mathrm{pb}} t$ is the electron beam plasma frequency. The magnetic field is along the $x$-axis and the beam is emitted at a finite angle to $B$. Figure 11a shows contours of the charge density of the electron beam and the spacecraft, which is modelled as a planar conductor. The helical primary beam can be seen for about two gyrations, embedded in beam electrons scattered by electrostatic fields from the beam space charge, plasma wave noise, and the spacecraft electric fields. Figure $11 \mathrm{~b}$ shows the ambient plasma electron density. The shaded regions are those with densities smaller than the unperturbed density. The depletion is brought about by the collection of return currents to the spacecraft as shown by the arrows. Behind the spacecraft this region is of a dimension perpendicular to $B$ which is comparable with the spacecraft dimension. In front of the spacecraft, the region is surrounding the primary beam 

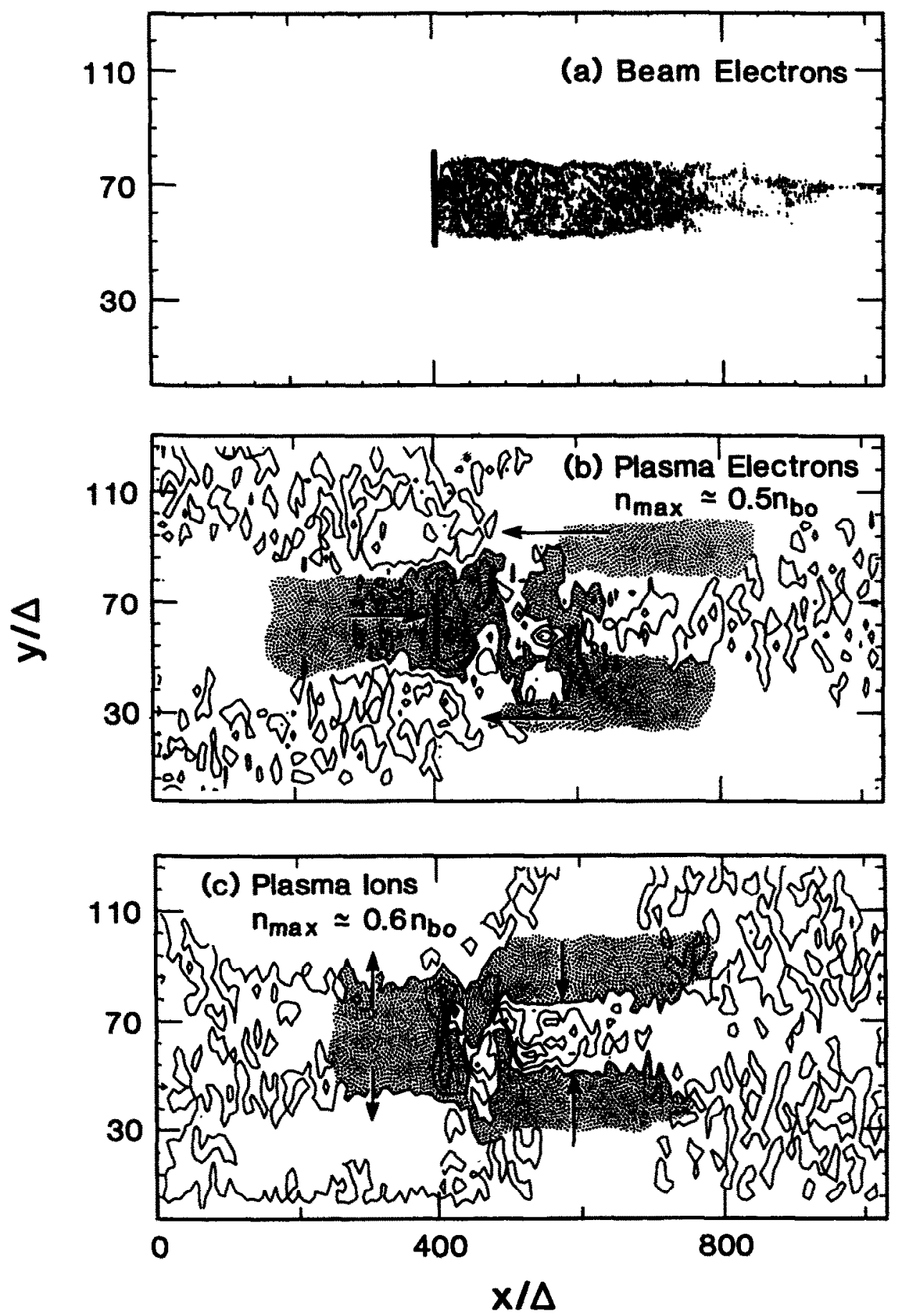

Fig. 11. Particle simulation of the injection of electron beams from spacecraft.

Contours of (a) the charge density of the beam and the spacecraft, (b) the plasma electron density, and (c) the plasma ion density at $\omega_{\mathrm{pb}} t=120$. Successive contours in (a) differ by a factor of 3.16 while those in (b) and (c) decrease linearly from the maximum value down to $8 \%$ of the maximum value. The arrows in (b) and (c) indicate the direction of the plasma flows in response to the beam injection. The shaded areas indicate the region where the density is smaller than in the initial plasma density (from Winglee and Pritchett, 1988). 
column. Current closure is in this simulation achieved by the plasma ion motion shown in Fig. 11c, which allows ions to flow across the magnetic field to a distance limited by their Larmor radius.

The beam structure obtained in the 2-D simulation, is consistent with optical observations of the electron beam structure during the STS-3 mission of the space shuttle (Banks and Raitt, 1988). It was found that the electron beam initially moved outwards along a classical helical trajectory. However, within a short distance electrons were deflected into paths parallel and anti-parallel to the magnetic field lines threading the primary helix. This resulted in the formation of a thin-walled, hot, electron torus with a radius equal to the primary beam gyroradius.

Winglee and Pritchett (1988) also found that a build-up of negative space-charge in the beam region close to the spacecraft may occur if the beam stagnation time $t_{\mathrm{s}}$ is smaller than the plasma response time $t_{\mathrm{rp}}$. For a given beam current, the ratio of the two time-scales depends on the beam density, the beam energy and the area of the spacecraft. The negative space-charge region is often referred to as a virtual cathode. The formation of a virtual cathode partially inhibits beam escape, destroys the spatial coherence of the beam, and creates additional plasma wave turbulence (Pritchett, 1990).

Early 1- and 2-D simulations gave rather restrictive values for the beam stagnation time and questions arose about beam escape from spacecraft in ionospheric beam experiments (Pritchett and Winglee, 1987; Winglee and Pritchett, 1988). However, other studies argued that experimental observations indicated beam escape, or at least that a substantial portion of the beam escaped the spacecraft (Neubert $e t$ al., 1988; Farrell, 1990). The discrepancy was resolved when 3-D simulations were performed. In three dimensions the criteria for beam escape are less restrictive because electrostatic fields decrease more rapidly with distance than in two dimensions. Simulations and experimental observations are now in agreement on this point : namely, that in most cases in past experiments, the beams were able to leave the payload environment (Okuda and Ashour-Abdallah, 1991; Pritchett, 1991).

Nevertheless, the virtual cathode effect is still important for electron beam emissions in space, and while it may not have limited the beam escape in past experiments, it certainly affects beam coherence. In addition, it is probably responsible for much of the electrostatic noise observed from the beam-emitting spacecraft both at low frequencies as observed in the SEPAC space shuttle experiment (Neubert et al., 1986; Cai et al., 1987) and at high frequencies as suggested by Neubert et al. (1991). Furthermore, return current electrons with energies in excess of the beam accelerator energy observed in the past (Managadze et al., 1988; Wilhelm et al., 1984; Waterman et al., 1988) and optical emissions (Sasaki et al., 1985a) are probably a result of such space-charge effects in the near-environment of the spacecraft rather than caused by a beam-plasma instability or a beam-plasma discharge.

Transient excursions of the spacecraft potential to levels well above the beam energy, often called "supercharging", have occasionally been observed (Sagdeev et al., 1981; Maehlum et al., 1988). It has been suggested that these high fields are associated with the formation of a virtual cathode. It is proposed that electrons are accelerated to high energies in the cathode region and that a percentage of these escape at energies which exceed the beam energy thereby driving the spacecraft to large positive potentials (Maehlum et al., 1988; Managadze et al., 1983, 1988; Denig et al., 1990).

The results on plasma heating, wave excitation, and beam dynamics can be summarized as :

(7) The 3-D structure of the disturbed electron beam region is now explored, revealing the dimension of the disturbed region to be about 4 beam electron Larmor radii perpendicular to the magnetic field. It has also been established that there exists a turbulent wake region behind the beam flux-tube.

(8) It is clear that transients in electrostatic potentials as well as return currents, azimuthal currents around the beam, and the primary beam current are important for the generation of broad-band radiation observed during electron beam ejection.

(9) Beam dynamics including electrostatic beam expansion, return current structure, and the phenomenon of virtual cathode and other space charge effects are now better understood.

(10) The success of the past decade in the understanding of the complete beam/spacecraft system is due to the synergy of detailed observations from multiple platforms, analytical models, and computer particle simulations.

\subsection{Neutral gas emissions}

The normal electrical neutralization of spacecraft at high altitudes relies solely on the ability to collect sufficient current from the ambient plasma since ionization of the neutral atmosphere provides little additional plasma. While the beam-system interactions mentioned previously have been thought to increase the electron current across the magnetic field, and thereby increase the return current through waveinduced particle diffusion (Linson, 1969 ; Hastings, 
1987), there are practical limits to the efficiency of such processes. Furthermore, while laboratory experiments indicate that such processes occur in this environment, it has not been possible to establish their importance in space experiments.

Another possibility for increasing the return current to a beam-emitting payload is through ionization of payload gases introduced accidentally through outgassing or deliberately, as in the case of attitude control thrusters. For example, in the $E C H O-4$ rocket experiment it was found that the injection of $\mathrm{N}_{2}$ gas by attitude control thrusters on the beam-emitting payload had the effect of lowering the spacecraft potential during beam emissions (Israelson and Winckler, 1979). This was also observed in the SEPAC experiment (Sasaki et al., 1985b) as well as in the CHARGE-2 experiment. In the case of CHARGE-2, $\mathrm{N}_{2}$ emissions from the beam-emitting mother as well as from the tethered, but passive daughter, reduced the spacecraft potential (Gilchrist et al., 1990; Banks et al., 1990). The reduction in potential is thought to be brought about by the ionization of the neutral gas cloud. The ionization is not dependent on the primary beam electrons but is triggered by return current electrons energized in the sheath. This conclusion was reached from the fact that the CHARGE2 daughter payload was located so far from the mother payload that the gas cloud could not have reached the beam region in the time it took the daughter to drop in potential. As a result of the decreased potential, wave turbulence was decreased (Neubert et al., 1991), and the beam escaped with a larger fraction of its energy as inferred from ground-based radar observations of the plasma density enhancement caused by BAI (Gilchrist, 1991). The reduction of spacecraft potential and easier escape of the beam from the payload in the presence of ambient or thruster neutral gases have also been found in computer simulations (Winglee, 1990).

Another means of controlling the spacecraft potential involves the use of the so-called plasma contactor, or hollow cathode. It operates by creating a dense plasma cloud in the vicinity of the spacecraft which then acts as a bridge between the more dilute background plasma and the spacecraft (Patterson, 1987; Williams et al., 1987; Katz and Davis, 1987). The plasma contactor in its normal mode of operation relies on the ionization within the hollow cathode of neutral gas flowing out from a pressure chamber. At positive bias potential, the contactor may operate in the so-called ignited mode in which the ionization occurs in the gas plume in the sheath region. Thus, the ignited mode is simply the ejection of neutral gas as observed in the CHARGE-2 experiment. The importance of this discovery is that with this method it is fairly simple and inexpensive to control large positive potentials and that such control seems to reduce plasma wave noise and allow the beam to escape with almost its complete accelerator energy. The limits in terms of beam current that can be neutralized in this way and the gas densities and flow rates required need to be explored in future experiments.

The results given above can be summarized:

(11) Large, positive payload potentials can be reduced by the ejection and subsequent ionization of neutral gas. The limits of beam currents that can be ejected during neutral gas emissions and the gas density and flow rates required to neutralize the payload need to be quantified with accurate experiments in space.

(12) When the payload potential is reduced by neutral gas emissions, the plasma wave noise is reduced and the beam escapes with a larger fraction of its accelerator energy.

\section{WHISTLER MODE RADIATION FROM ELECTRON BEAMS}

\subsection{Background}

We now explore some basic features of wave generation from pulsed and d.c. electron beam experiments in space plasmas. We pay particular attention to whistler wave generation in ionospheric plasmas and to the limits on the electron beam energy for wave generation in the two principal resonances, Landau resonance and cyclotron resonance. The "standard" way of calculating the radiation from a single particle spiralling in a magnetized cold background plasma is to solve Maxwell's equations with the moving particle as the current source (Mansfield, 1967; McKenzie, 1967). In this treatment, the radiation is found to be particularly intense for waves satisfying the resonance condition:

$$
R=s \omega_{c}^{*}+k_{1} v_{1}-\omega=0 .
$$

In equation (13), $\omega_{\mathrm{c}}^{*}$ is the relativistic beam particle cyclotron frequency:

$$
\omega_{c}^{*}=\omega_{c}\left(1-v^{2} / c^{2}\right)^{1 / 2},
$$

where $v$ is the particle velocity and $c$ is the velocity of light. The component of the wave vector parallel to the magnetic field, $k_{1}$, is given by

$$
k_{1}=k \cos \theta
$$

where $k$ is the wavenumber and $\theta$ the angle of the wavenumber to the magnetic field. Similarly, for the particle velocity along the magnetic field: 


$$
v_{1}=v \cos \alpha,
$$

where $\alpha$ is the pitch angle of the particle. The wave frequency $\omega$ is related to $k$ through the plasma dispersion relation.

The parameter $s$ takes on the value 0 for Landau resonance where Cherenkov radiation is emitted, 1 for cyclotron resonance, and -1 for anomalous cyclotron resonance. Larger integer values denote higher order cyclotron resonances. Condition (13) is often referred to as the first-order resonance. Since the background plasma is usually inhomogeneous, waves and particles in resonance at one point will become offresonant as they propagate away to other regions. Enhanced interaction is achieved when waves and particles are also in second-order resonance, namely when :

$$
\mathrm{d} R(z) / \mathrm{d} z=0,
$$

where $z$ is the coordinate along the background magnetic field (Neubert et al., 1987).

As an example, we consider the case of whistler mode radiation. An approximate expression for the refractive index $\mu$ for $\omega>\omega_{\mathrm{LHR}}$ and $\omega_{\mathrm{ce}}<\omega_{\mathrm{pe}}$ is :

$$
\mu=\omega_{\mathrm{pe}} /\left[\omega\left(\omega_{\mathrm{ce}} \cos \theta-\omega\right)\right]^{1 / 2},
$$

where $\mu=k c / \omega, \omega_{\mathrm{pe}}$ is the electron plasma frequency, and $\omega_{c e}$ the electron gyrofrequency. The resonance cone angle is the angle at which the refractive index and the wavenumber goes to infinity:

$$
\cos \theta_{\mathrm{r}}=\omega / \omega_{\mathrm{ce}} \text {. }
$$

Real solutions to equation (18) require $\theta<\theta_{r}$. Figure 12 shows a sketch of the whistler mode refractive index surface as function of $\theta$ for two values of the wave frequency. Also shown is the direction of the group velocity, which is perpendicular to the surface.

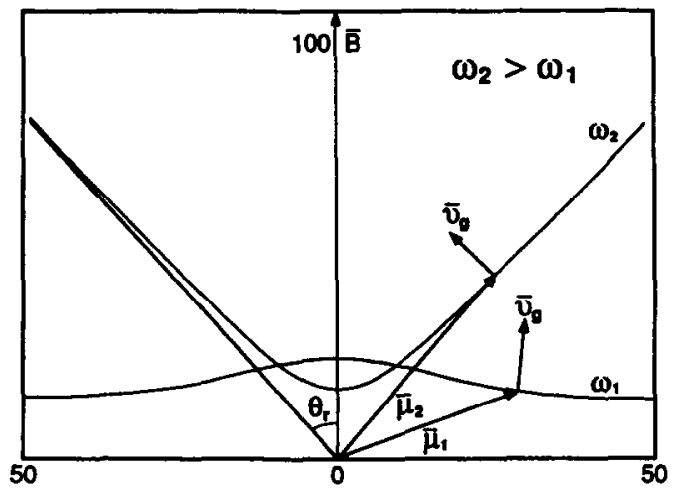

Fig. 12. THE WHISTLER MODE REFRACTIVE INDEX SURFACE. The arrows indicate the direction of the group velocity, which is perpendicular to the surface.
For $\omega<\omega_{c e} / 2$ (satisfied by $\omega_{1}$ in Fig. 12) the refractive index surface has a dimple around $\theta=0$ such that a minimum in $\mu_{1}$ occurs for $\theta=\theta_{g}$, the so-called Gendrin angle. For $\omega>\omega_{\mathrm{ce}} / 2$ the dimple disappears (as is the case for $\omega_{2}$ in Fig. 12) and the minimum in $\mu_{1}$ occurs for $\theta=0$. The minimum value of $\mu_{1}$ found from equation (18) is :

$$
\mu_{\| \min }=2 \omega_{\mathrm{pe}} / \omega_{c e} \quad\left(\omega<\omega_{c e}\right)
$$

and the Gendrin angle is :

$$
\cos \theta_{\mathrm{g}}=2 \omega / \omega_{\infty} \quad\left(\omega<\omega_{\infty} / 2\right) .
$$

The relativistic expression for the electron energy, $E$, normalized to the electron rest mass $(511 \mathrm{keV})$ is :

$$
E=\left(1-v^{2} / c^{2}\right)^{-1 / 2}-1,
$$

where the resonance beam electron velocity found from equation (13) is :

$$
v_{1} / c=g_{3}^{*} / \mu_{1}
$$

with $g_{s}^{*}=1-s \omega_{c}^{*} / \omega$.

From equation (23) it is seen that at a given wave frequency there is a maximum beam energy for which resonance is possible. This energy corresponds to the minimum in $\mu_{1}$. A minimum of the beam energy can be found by noting that small wavelength whistler waves have a very large refractive index and are primarily electrostatic in nature. As a result they are strongly damped as they propagate through a plasma. Using a wavelength of $1 \mathrm{~m}$ as a practical lower limit for whistler mode propagation, this corresponds to a maximum of $k=2 \pi \mathrm{m}^{-1}$.

The maximum and minimum electron energies for Landau resonance are shown in Fig. 13 and for cyclo-

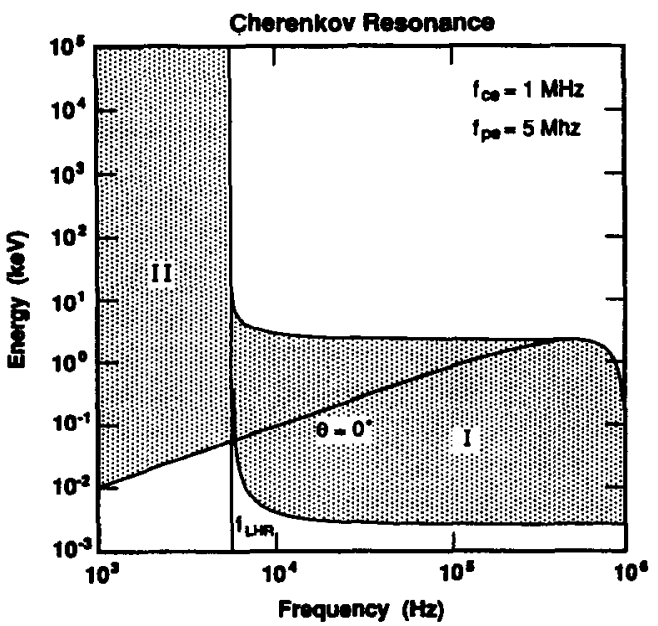

Fig. 13. MAXimum AND Minimum Resonance ENERGY For CherenKov resonance.

Shaded areas indicate regions of resonance. 
tron resonance in Fig. 14. The region allowing resonance with whistler mode waves is marked with $I$. (Region II is discussed later.) Also shown is a curve through this region which corresponds to the resonance energy for $\theta=0^{\circ}$. It is assumed that $\omega_{c e} / 2 \pi$ and $\omega_{\mathrm{pe}} / 2 \pi$ are $10^{6} \mathrm{~s}^{-1}$ and $5 \times 10^{6} \mathrm{~s}^{-1}$, respectively, which are values representative for the peak of the ionosphere. The ion population is exclusively $\mathrm{O}^{+}$. The figures show the resonance energies according to the Stix (1962) formulation of the cold plasma dispersion relation and includes relativistic effects on the beam electrons. It is assumed that the pitch angle, $\alpha=0^{\circ}$.

At frequencies below one half the electron gyrofrequency and above the lower hybrid frequency, $f_{\text {LHR }}$, the maximum energy for Landau resonance is approximately constant in wave frequency for given background plasma parameters [from equations (20) and (23)]. In the ionosphere the ambient plasma density and therefore the plasma frequencies may have large fluctuations depending on local time, geographic location, and even small variations in altitude. As a result, the maximum energy for Landau resonance may also vary and will generally increase with decreasing density. Many electron beam experiments performed in the past have been in the range from 1 to 5 $\mathrm{keV}$ and were therefore expected to generate waves through Landau resonance in the whistler mode. Some experiments, in particular the ECHO series, used higher energies, up to $40 \mathrm{keV}$, and the degree to which Cherenkov radiation was generated via Landau resonance depended on the spacecraft altitude and ionospheric conditions.

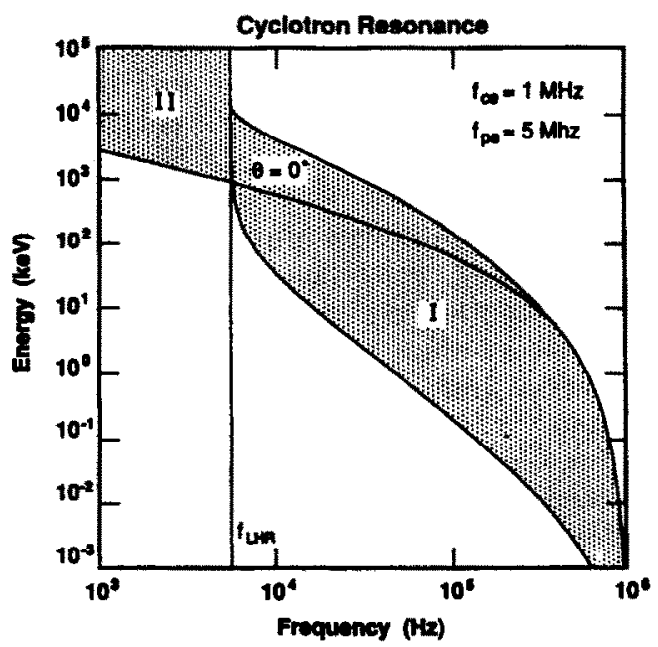

Fig. 14. Maximum aND MINMUM RESONANCE ENERGY fOR CYCLOTRON RESONANCE.

Shaded areas indicate regions of resonance.
For low frequency waves in cyclotron resonance, $g$, becomes large and, consequently, the resonance energy becomes large, as can be seen from equation (23). As the wave frequency approaches the lower hybrid frequency, the resonance cone angle approaches $90^{\circ}$ and the maximum as well as the minimum resonance particle energy go to infinity. Because of the higher energy required for cyclotron resonance, this resonance has not been studied to the same degree as Cherenkov resonance. Future experiments with higher energy accelerators may study cyclotron resonance. It will be relatively simple to distinguish between cyclotron and Landau resonance. In the latter, the wave energy propagates in the same direction as the beam electrons, while in the former the waves propagate opposite to the beam electrons.

Below the lower hybrid resonance the topology of the wave normal surface changes. The resonance cone disappears and the wave normal surface becomes closed. The maximum in energy found for frequencies above the lower hybrid resonance becomes a minimum in energy below the lower hybrid resonance. The region of electron energies in resonance here is marked II in Figs 13 and 14.

\subsection{Radiation from d.c. electron beams}

On auroral field lines charged particles are accelerated by localized electric fields thought to be located at altitudes of 2-4 Earth radii. The result is the creation of an electron plasma with beam-like distributions in velocity. These electrons excite aurora as they penetrate the upper atmosphere. They also generate plasma waves over a wide frequency range. Figure 15 shows a typical frequency spectrum of the electric field intensity observed by the $D E 1$ satellite as it traverses the auroral oval from the polar cap and to the plasmasphere (Gurnett et al., 1983). Above the electron gyrofrequency auroral kilometric radiation (AKR) and emissions at the upper hybrid frequency are seen, while below the gyrofrequency, in the whistler mode frequency range, auroral hiss and plasmaspheric hiss are seen as the spacecraft enters the plasmasphere. In particular we point out the funnel shape of the auroral hiss as it appears on a frequency-time plot as shown in Fig. 15. This shape is consistent with the idea that the whistler wave noise is generated with wave normals close to the resonance cone through Landau resonance with energetic auroral electrons accelerated at some localized altitude below the spacecraft.

The funnel shape is a result of the propagation characteristics of whistler mode waves at the resonance cone. The higher the wave frequency, the narrower the resonance cone; because the direction of 
the group velocity for waves at the resonance cone is perpendicular to $k$, the angle of propagation to $B$ increases with wave frequency. Thus high-frequency emissions are first encountered as the spacecraft approaches the field line. In the case of a point source, only a V-shaped outline of the funnel will be seen. Such emissions are frequently observed and have been named "saucers" (James, 1976). A "filled-in" funnel requires a beam (or sheath) source which extends from a given point in space.

Emissions of a similar nature have been observed in artificial beam experiments. In the Spacelab-2 experiments made with the space shuttle, a small satellite, the Plasma Diagnostics Package (PDP), was released from the shuttle and separated to distances of about $300 \mathrm{~m}$ during a $6 \mathrm{~h}$ period. During that time, the shuttle was maneuvered to provide four magnetic conjunctions of the PDP and the shuttle. During two of these, an electron beam (with electron energies of 1 $\mathrm{keV}$, and currents of 50,100 , or $150 \mathrm{~mA}$ ) was emitted from the shuttle. The electric field intensity observed by the $P D P$ during the ejection of a continuous beam at $50 \mathrm{~mA}$ is shown in Fig. 16 (Gurnett et al., 1986). Over frequencies between about $30 \mathrm{kHz}$ and up to the electron cyclotron frequency a funnel-shaped emission is seen which bears close resemblance to the auroral hiss shown in Fig. 15. As the PDP approaches the beam flux-tube the high frequency emissions are observed first followed by lower frequency emissions. When the $P D P$ crosses the beam, emissions are also observed close to the local upper hybrid frequency inside the beam.

The similarities of the natural and beam-generated emission led to the conclusion that they were a result of the same process. In the case of the Spacelab-2 beam, it was found that the spectral shape on timefrequency plots as well as the electric field polarization were consistent with the assumption of Landau resonance between beam electrons and whistler waves at the resonance cone (Farrell et al., 1988).

The Spacelab-2 observations allowed the quantitative comparison of theories for wave generation by electron beams with the observations. Studies in the past of naturally occurring plasma waves and particle distributions using the formulation of Mansfield (1967) and McKenzie (1967) led to the conclusion that incoherent Cherenkov radiation (the radiation from each electron is added incoherently) was several orders of magnitude lower than observed (Taylor and Shawhan, 1974). Because the power radiated by coherent radiation is proportional to $N^{2}$, where $N$ is the number of particles, while the power from incoherent radiation is proportional to $N$, an assumption of some coherency will increase the power and bring it in agreement with observations. Similar conclusions were reached for the Spacelab-2 electron beam (Farrell et al., 1988). It was found that the radiation from the beam was orders of magnitude above expectations for an incoherent process and that some coherency must have been present. Following an idea of Bell (1968), analytical calculations and 1-D computer simulations were presented by Farrell et al. (1989), which demonstrated that density perturbations in the electron beam density, caused by Langmuir oscillations in the beam, as observed during magnetic conjunction, can provide the necessary coherence. The density perturbations were found to propagate with a velocity close to the beam electron velocity and could therefore radiate through the Landau resonance.

Later simulation studies in two dimensions confirmed this conclusion with minor modifications. Pritchett et al. (1989) found that the beam-plasma interaction creates a current structure that radiates much like an antenna through the Cherenkov radiation mechanism. In their study, the beam region carried no net current at the start of the simulation. This was achieved by giving the higher density background plasma an appropriate low energy drift velocity in the opposite direction to the beam velocity. Although the average net current is zero, radiation is still generated because of the electron current perturbations created in the beam by micro-instabilities.

Other studies of electron beam radiation include that of Wong and Lin (1990) which presents an analytical model for the modes generated inside the beam and the coupling at the beam edge of these modes to the ambient plasma. It was found that this process could generate whistler mode radiation which propagates in the ambient plasma with $k$ close to the resonance cone as observed in the Spacelab-2 experiments. A particle simulation of the Spacelab-2 experimental results was presented in Omura and Matsumoto (1988). The simulation was done in two dimensions with periodic boundary conditions in the dimension along the beam. Such boundary conditions amount to simulating an infinite beam. Even so, the results showed wave spectra as a function of distance from the beam which resembled the funnel-shaped spectra of Spacelab-2. They suggested that the funnel was a result of frequency-dependent damping as the waves propagate away from the beam. Furthermore, they found that the wavelength of the electrostatic noise generated inside the beam column did not match the wavelength of the radiated whistler wave noise and concluded that the radiation was not caused by bunching of the beam electrons.

The results of radiation from continuous electron beams can be summarized as follows: 


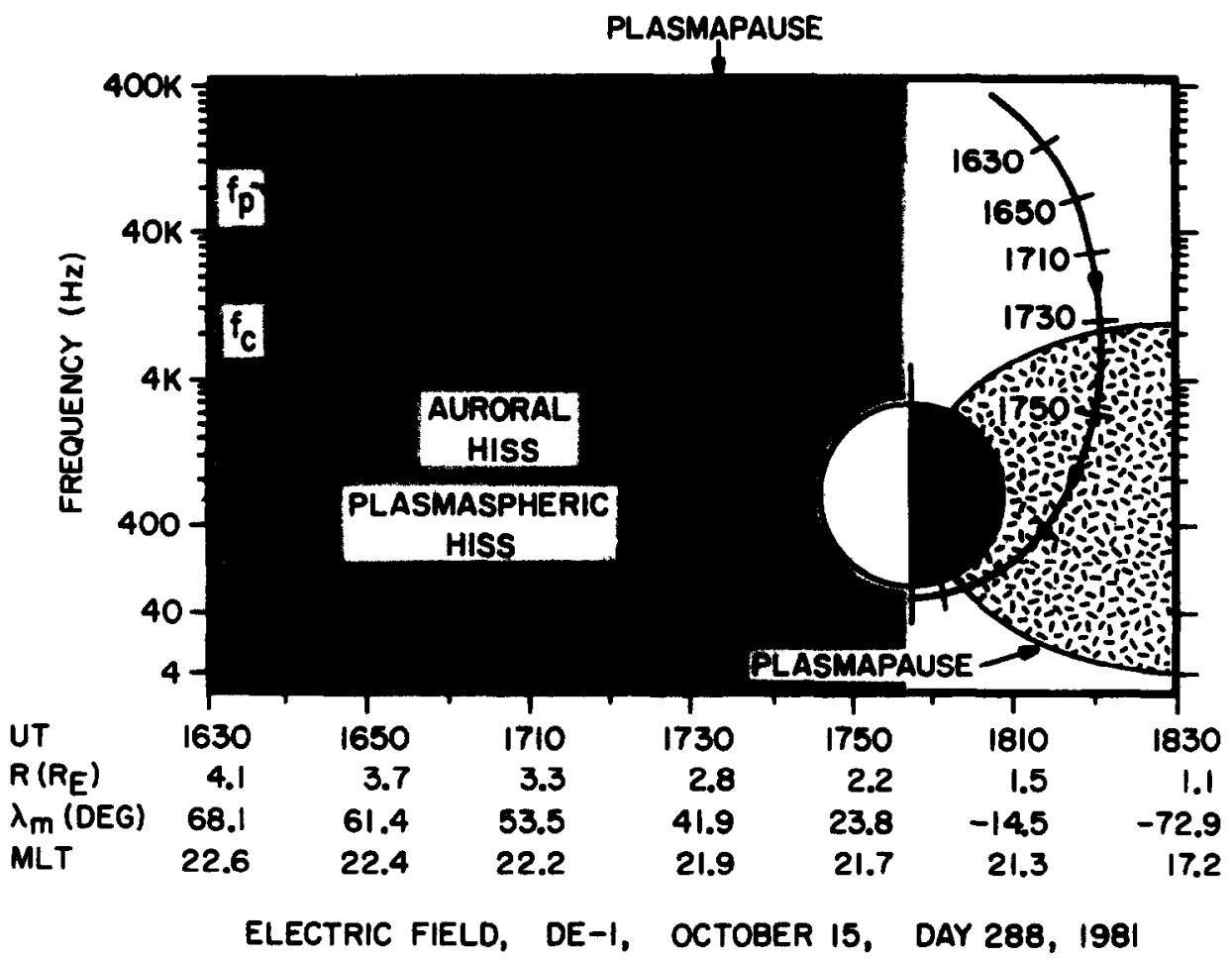

Fig. 15. A REPRESENTATIVE SPECTROGRAM OF THE ELECTRIC FIELD INTENSTTIES FOR A NIGHT-SIDE CROSSING OF THE AURORAL FIELD LINES AS OBSERVED BY DE-1 (FROM GURNETT et al., 1983). 


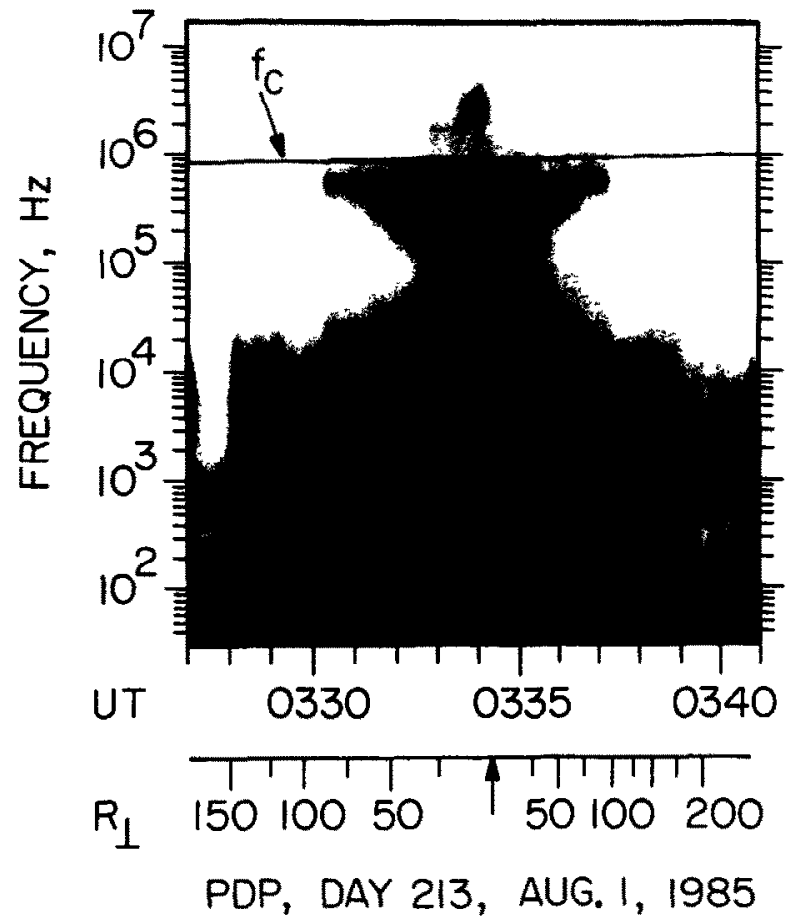

Fig. 16. A SPECTROGRAM OF THE ELECTRIC FIELD INTENSITY DURING ELECTRON BEAM EJECTIONS FROM THE SPACE SHUTTLE.

The free-fiying $P D P$ is in magnetic conjunction with the shuttle and in the beam region at the time indicated by the arrow (from Gurnett et al., 1986). 


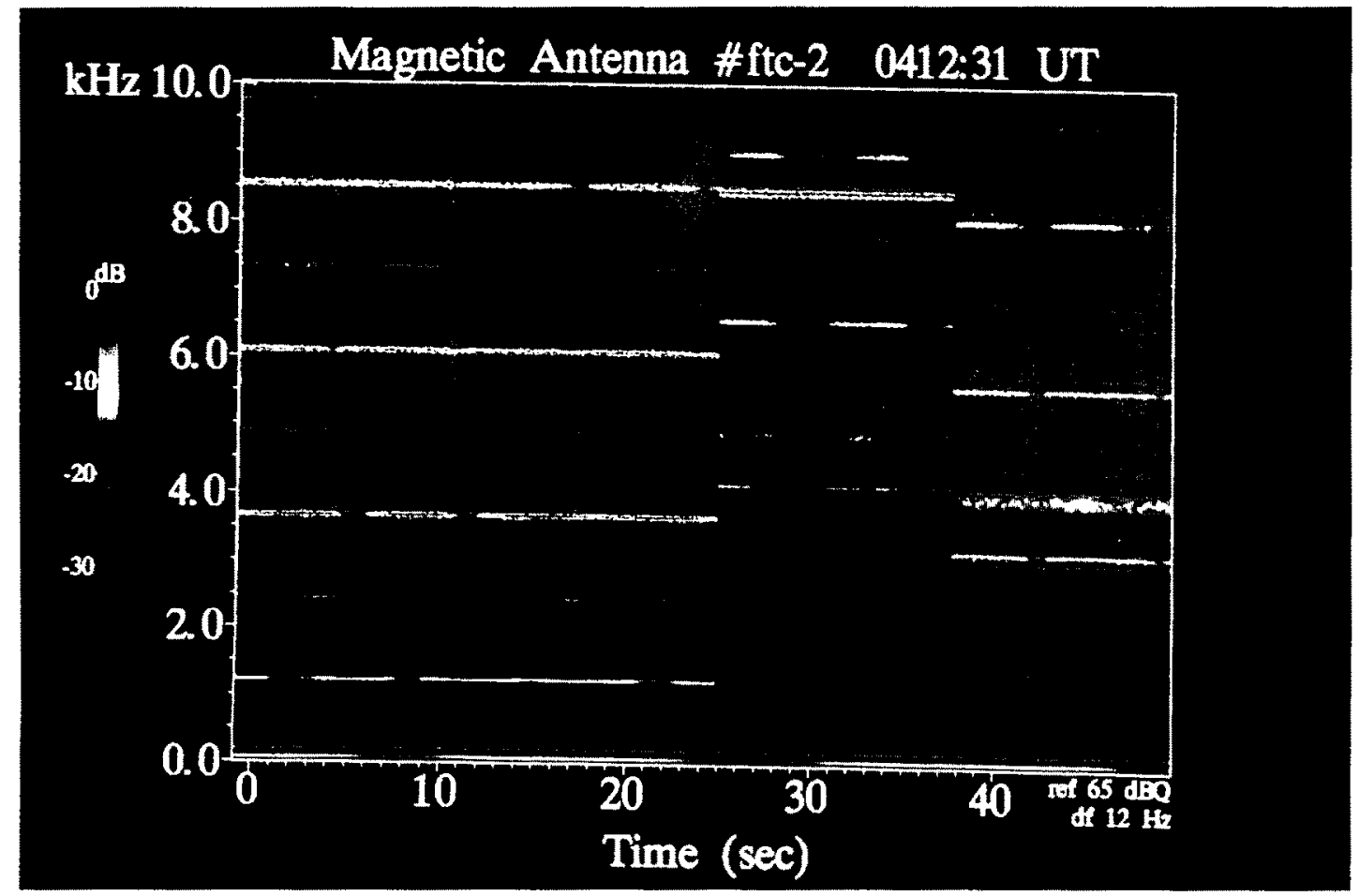

Fig. 17. The MAGNETIC FIELD IN THE FREQUENCY RANGES 0-10, 20-10, AND 20-30 kHz AS A FUNCTION OF TIME.

The $P D P$ is free-flying and the FPEG is pulsed at $1.22 \mathrm{kHz}$ (from Bush $e t$ al., 1987 ; Neubert et al., 1988). 
(13) The consensus from experiments and computer simulations is that in past experiments, employing kiloelectronvolt energy electron beams, whistler wave radiation is generated through Cherenkov resonance with beam electrons.

(14) The intensity of the radiation is between the levels expected for incoherent and coherent radiation. Micro-instabilities in the beam are found to be important for the establishment of quasi-coherency and thereby for the creation of the structure of the radiating current.

(15) The details of the formation of the radiating current structure are not well understood. It is thought that Langmuir oscillations play an important role in bunching the electrons. Spacecraft potential oscillations may also be important.

\subsection{Radiation from pulsed electron beams}

A pulsed electron beam generates electromagnetic radiation at the pulsing frequency and its harmonics. The radiation signature is therefore easier to distinguish from ambient plasma noise than the radiation from continuous beams. Furthermore, the spectrum of the radiation contains information on the spatial structure of the pulsed beam which can be compared with the format of the injected beam. In this way additional detailed information on beam dynamics and radiation can be obtained. Since the very first space-borne electron beam experiment (Hess et al., 1971), it has been conjectured that pulsed electron beams could be used as virtual antennas for communication purposes, for instance between space and ground or between two platforms in space. Beams have an advantage over conventional electric dipole antennas or magnetic dipole loop antennas, which is particularly important in the VLF whistler mode frequency range; because of the large wavelength of VLF waves it takes very long electric dipoles or large loops to efficiently radiate VLF waves.

The STS-3 mission of the space shuttle orbiter, flown in 1982, carried an electron beam accelerator especially designed to study the radiation from pulsed electron beams. The accelerator, the Fast Pulse Electron Generator (FPEG) emitted 1 keV energy electron beams with currents of 50,100 or $150 \mathrm{~mA}$. The beam could be square-wave modulated from d.c. and up to several hundred kilohertz with a pulse rise-time of the order of $10^{-7} \mathrm{~s}$ (Banks et al., 1987). The electromagnetic radiation generated by beams pulsed at ELF and VLF frequencies was observed with wave receivers mounted on a $P D P$, which was either stored in the payload bay, or mounted on the Remote Manipulating System (RMS) (Shawhan et al., 1984; Reeves et al, 1988b).
Attempts were made to detect the radiation at remote locations in the magnetosphere by executing beam emission sequences coordinated with the $D E 1$ satellite at times when the shuttle and the satellite were in magnetic conjunction. The results were negative; however it was later shown that either the STS-3 attitude was such that the electrons hit the main body of the orbiter, or raytracings showed that it was not possible for whistler mode waves to propagate from the STS-3 location up to the vicinity of the $D E 1$ satellite (Inan et al., 1984). The FPEG/PDP was flown again in the Spacelab-2 mission of 1985 as mentioned earlier. Radiation was observed on the $P D P$ freeflyer out to its maximum separation distance of about $300 \mathrm{~m}$ (Bush et al., 1987; Reeves et al., 1988a ; Neubert et al., 1988). Again no radiation was observed on the ground or on $D E 1$.

Results from other experiments studying the radiation characteristics of pulsed electron beam emissions are reported in Cartwright and Kellogg (1974); Reme (1980); Holzworth and Koons (1981); Winckler et al. (1984, 1985); Kellogg et al. (1986); Sasaki et al. (1987, 1988); Goerke et al. (1990); Neubert et al. (1991). In most of these experiments, attempts were made to detect the wave fields on the ground. The results to date have proved negative, and the largest distance from the beam source that VLF wave fields have been observed is of the order of 1-2 $\mathrm{km}$.

In parallel with the experimental efforts involving the FPEG, a theory for the radiation from the squarewave modulated electron beam was developed. The first model considered the coherent radiation from a finite train of pulses injected into a magnetized plasma (Harker and Banks, 1983). For simplicity, each pulse was assumed to traverse an unbounded path length $(-\infty$ to $+\infty)$. The beam was assumed to radiate coherently and the radiation was determined for the frequency range from the lower hybrid frequency to the electron gyrofrequency. In Harker and Banks (1985) the power radiated by a beam propagating into a half space $(0$ to $+\infty)$ was calculated for frequencies both below and above the lower hybrid frequency. The power was found for the far-field region, which is the region at distances beyond a few perpendicular wavelengths from the beam source. Since the Space$l a b-2$ observations were performed in the near-field region a near-field model was developed (Harker and Banks, 1987) giving the complete polarization of the electric field. Observations showed, however, that associated with the pulsed beam injection, considerable broad-band electrostatic noise was generated just as for the case of continuous beam injection. This made it difficult at times to determine the 
spectral component on the pulsing frequency or its harmonics. The magnetic field, on the other hand, was very clear and undisturbed from broad-band noise. The calculations were therefore extended to include the near-field components of the magnetic field (Neubert and Harker, 1988). Finally, the theory has been extended to allow calculations of signal levels on the ground from pulsed beam emissions in space (Harker et al., 1991).

The theory of Harker and Banks is based on the work of McKenzie (1967). It assumes that the radiating current is that of an infinitely thin electron beam spiralling around the geomagnetic field under the influence of the Lorentz force. The beam is squarewave modulated (pulsed) and the beam electrons within a pulse are assumed to radiate coherently. Effects of electrostatic expansion of the helical beam, return currents and other processes which destroy the ideal beam structure are accounted for through an attenuation factor which assumes that the ability of the beam to radiate coherently decreases exponentially with distance from the beam accelerator.

The Harker and Banks theory was developed particularly for the square-wave modulated electron beam of the FPEG. Other treatments include the radiation from pulsed electron beams at zero degree pitch angle (Lavergnat and Lehner, 1984; Ohnuki and Adachi, 1984) and sinusoidally modulated electron beams at arbitrary pitch angles (Lavergnat et al., 1984).

As a result of the assumption of a square-wave modulated current source, the radiated fields contain a factor $D$, the so-called duty cycle factor:

$$
D=1 / \eta \sin (\eta \pi b / d),
$$

where $b / d$ is the duty cycle, $b$ is the on-time for a pulse, and $d$ is the total modulation period. The parameter $\eta$ is the harmonic number. The factor $D$ arises naturally from the Fourier transform of a square-wave function.

During the Spacelab-2 experiment, several predictions of the Harker and Banks theory were tested. The $P D P$ free-flight provided observations of the electric and magnetic fields during a beam sequence in which the beam $(100 \mathrm{~mA})$ was pulsed with $50 \%$ duty cycle at $1.22 \mathrm{kHz}$. The $P D P$ was located during this beam sequence at distances from a few meters to $150 \mathrm{~m}$ from the electron beam. A spectrogram of the magnetic field is shown in Fig. 17 (Bush et al., 1987; Neubert $e t$ al., 1988). The relative signal intensity is color coded showing the fundamental and the odd harmonics of the modulation frequency as horizontal lines. In the first half of the panel $(0-25 \mathrm{~s})$ the frequency band is from 0 to $10 \mathrm{kHz}$, the following quarter
$(25-38 \mathrm{~s})$, the band is $20-10 \mathrm{kHz}$ with $20 \mathrm{kHz}$ at the bottom of the frequency scale, and in the last quarter the band is from 20 to $30 \mathrm{kHz}$. During the time interval shown the distance of the $P D P$ was $75 \mathrm{~m}$ perpendicular from the beam.

The Fourier transform of a square-wave function with a $50 \%$ duty cycle has vanishing even harmonics and odd harmonics with amplitudes that varies as $1 / f$. Figure 18 shows the magnetic field amplitude of the narrow-band emissions observed at three different locations of the $P D P$ relative to the beam (Reeves et al., 1988a). As can be seen, the amplitude does decrease approximately as $1 / f$. Note also that even harmonics are present, although weaker by an order of magnitude. This indicates that the beam is losing some of its square-wave form within the $300 \mathrm{~m}$ distance of the $P D P$ and the shuttle. However, as pointed out by Reeves et al. (1990b), the duty cycle factor is very sensitive to changes in the duty cycle. A change from 50 to $50.5 \%$ is enough to account for the amplitude of the even harmonics.

The beam resonance condition determines $k_{1}$. The dispersion relation has two roots or two values of $k_{\perp}$ for given values of $k_{\boldsymbol{l}}$ and $\omega$. In certain frequency bands one of these roots becomes imaginary, which corresponds to evanescent waves. In the near-field these waves can give important contributions to the electromagnetic field. In the theory of Harker and Banks root 1 is evanescent below $f_{\text {LHR }}$ while root 2 is continuous across $f_{\text {LHR }}$. Above $f_{\text {LHR }}$ rootl corresponds to waves generated at the resonance cone, while root 2 corresponds to waves generated in the central hump of the refractive index surface. Studies of the amplitude of the harmonics as a function of distance from the beam show that the signal amplitude roughly follows the predictions for the root 2 waves. For the Spacelab-2 free-flight, $f_{\mathrm{LHR}}$ was around $3 \mathrm{kHz}$, and therefore the first harmonic was below $f_{\text {LHR }}$ while the third and higher harmonics were above $f_{\text {LHR }}$. The root 1 solutions for the higher harmonics have, according to the theory, much larger amplitudes than observed. Furthermore, the first harmonic (which is evanescent for root1) should be strong very close to the beam, and decrease rapidly with distance until the root 2 waves become dominant. This behavior was not observed in the magnetic field data. Rather, the amplitude varied with distance much as predicted for root 2 waves (Reeves et al., 1990a).

As shown in Fig. 19, the first harmonic of the electric field below $f_{\text {LHR }}$ did decrease relatively rapidly with distance (Reeves et al., 1988a). At the same time, emissions around the second and third harmonics, broad-band as well as narrow-band, became relatively dominant. It is possible that these waves are lower 


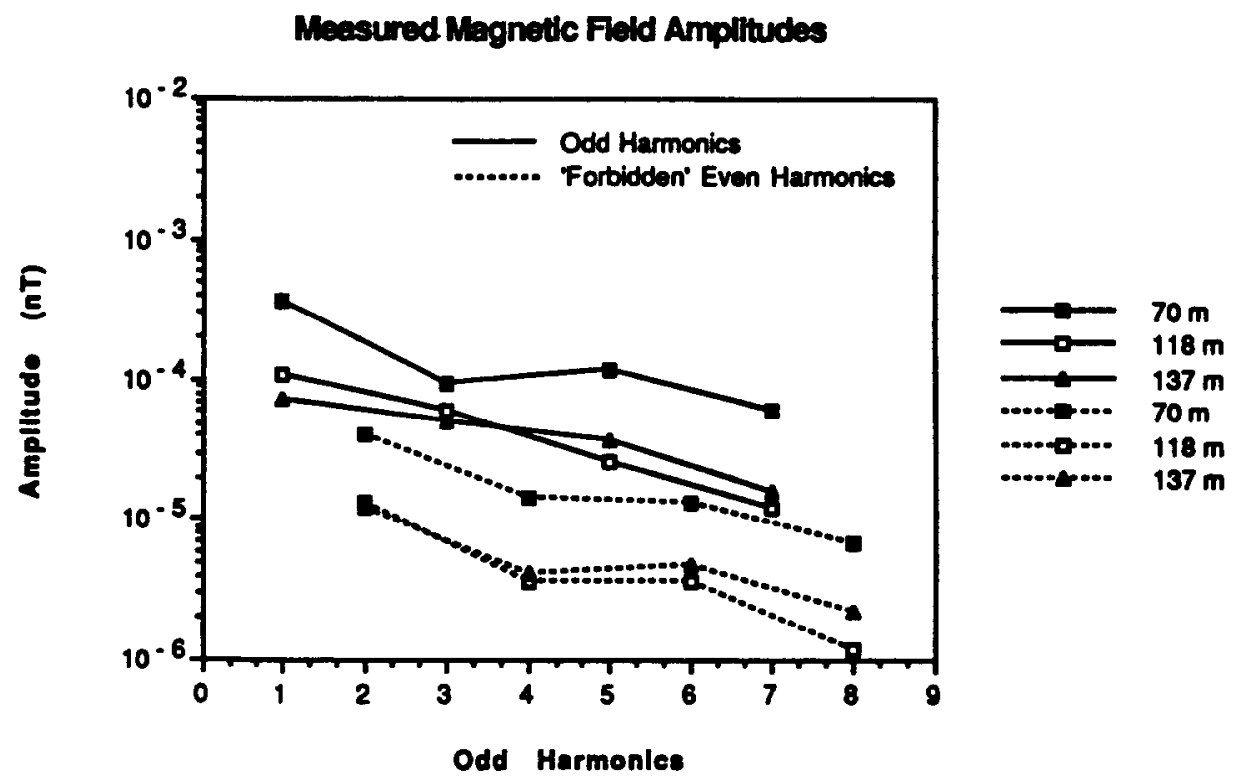

Fig. 18. MAGNETIC FIELD AMPLITUDE OF THE HARMONICS OF THE BEAM PULSING FREQUENCY $(1.22 \mathrm{kHz})$ AT THREE DIFFERENT LOCATIONS OF THE PDP RELATIVE TO THE BEAM (FROM REEVES et al., 1988a).

hybrid waves as suggested by computer particle simulations (Matsumoto and Fukuchi, 1985; Hwang and Okuda, 1989).

The dependence of the wave field amplitude on the duty cycle has been studied by injecting a sequence of pulses with constant pulsing frequency and varying the duty cycle. Figure 20 shows the magnetic field amplitude observed in the payload bay of the first

\section{Measured Electric Fild Amplitude:}

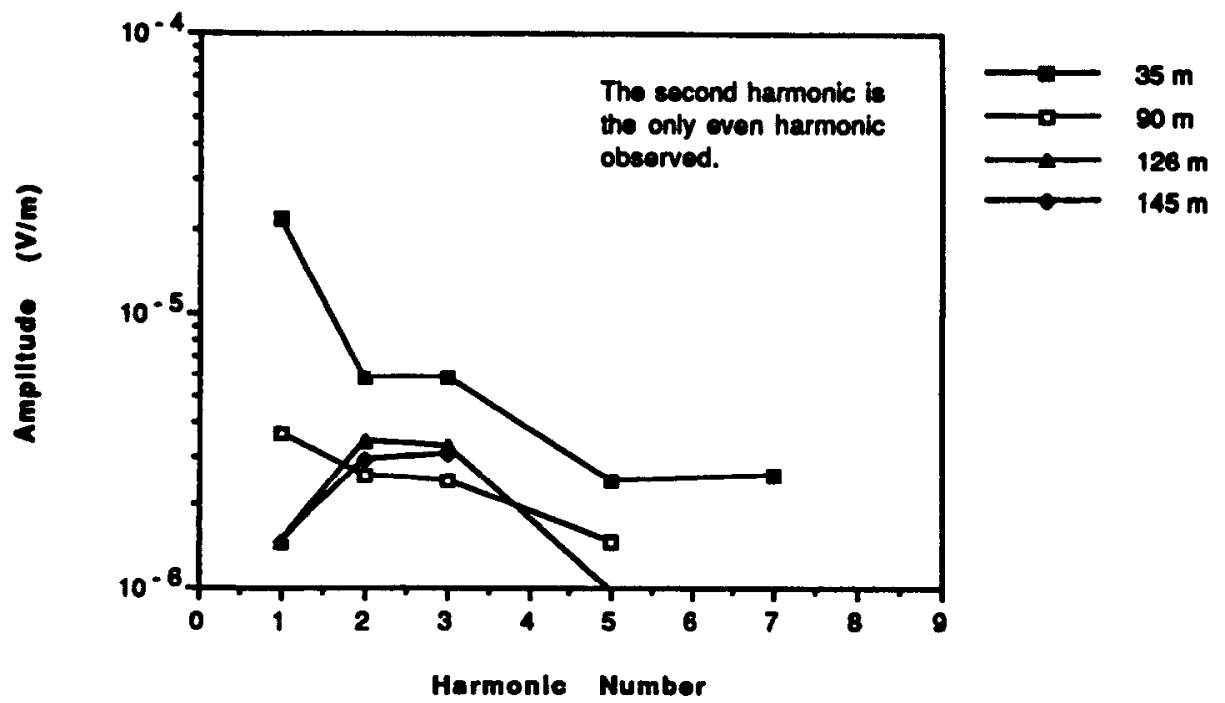

FiG. 19. ELECTRIC FIELD AMPLITUDE OF THE HARMONICS OF THE BEAM PULSING FREQUENCY $(1.22 \mathrm{kHz})$ AT DIFFERENT LOCATIONS OF THE PDP (PROM REEVES et al., 1988a). 


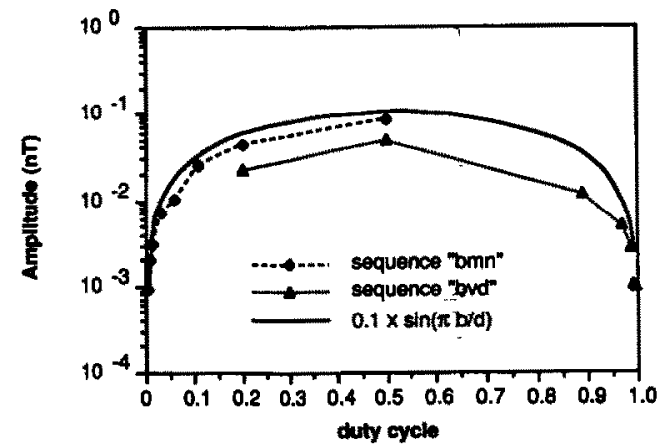

FIG. 20. MAGNETIC FIELD AMPLITUDE OF THE FUNDAMENTAL FREQUENCY AS A FUNCTION OF DUTY CYCLE.

The solid line is the duty cycle factor $D$ arbitrarily normalized to 0.1 (from Reeves et al., 1990b).

harmonic as a function of duty cycle (Reeves et al., $1990 \mathrm{~b}$ ). The factor $D$ is normalized to the arbitrary value of 0.1 in order to enable the comparison with the observations. As can be seen, the agreement between observations and predictions is quite good. As the duty cycle goes to zero, the average current goes to zero and the field amplitude approaches some small background level. For the duty cycle approaching $100 \%$, the beam becomes continuous and only broadband noise is generated. Here, the amplitude again approaches a very small value. One question that has remained unanswered in the past has been whether the sudden onset and turn-off of the beam, that is, the leading or trailing edge of the beam or sudden changes in the spacecraft potential were responsible for the radiation. In this case, radiation should be independent of the duty cycle. Thus, the Spacelab-2 study shows that the source at least to some approximation is square-wave modulated as predicted by theory.

The polarization of the beam-generated fields was studied in Neubert et al. (1990c). Although only one component of the electric and one component of the magnetic field was measured, the spin of the $P D P$ was utilized to find the polarization of the fields in the spin plane. It was found that the observed polarization did not agree well with the predicted polarization and that therefore the current structure departed from the helical structure assumed in the model. Another possibility comes from the fact that the theory presented in Harker and Banks (1987) neglects the contribution from a branch cut in the integration over $k_{\perp}$ which corresponds to ray propagation, and only considers the simple poles which correspond to surface waves. The model is, therefore, incomplete as it stands.

A final comparison with theory is shown in Fig. 21 (Reeves et al., 1990b). The amplitude of the fun-

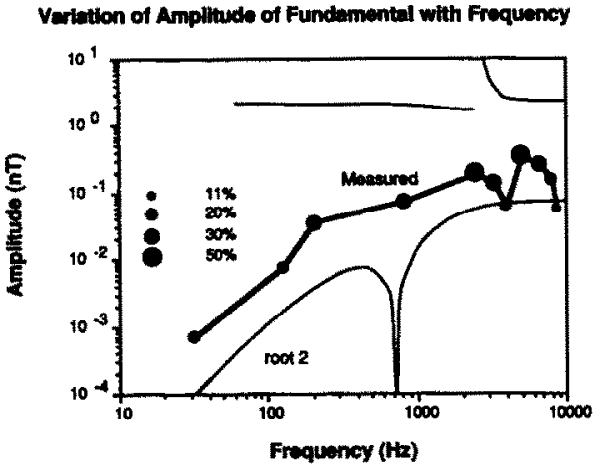

Fig. 21. The magnetic field ampltudde of the funDAMENTAL AS A FUNCTION OF THE PULSING FREQUENCY. Predicted curves are shown for Cherenkov rootl and root2, The size of the markers indicate the duty cycle (from Reeves et al., 1990b).

damental component is plotted as function of frequency. This coverage in frequency required a compromise on the duty cycle. The duty cycle used is shown by the size of the markers, $50 \%$ duty cycle having the largest marker. At this time the $P D P$ was located in the payload bay. Also shown are the predictions of the model for rootl and root2. Again it can be concluded that the model predictions for root 2 are in general agreement with observations while root 1 predicts amplitudes many orders of magnitude above observations.

The results from pulsed electron beam studies can be summarized as follows :

(16) Observations show that the spectral structure of the wave emissions closely follows the spectral structure of the injected beam current. A square-wave modulated beam generates harmonics as expected from the Fourier transform of a square-wave function.

(17) A closer comparison with the theory of Harker and Banks (1987) reveals that the amplitude dependence of individual harmonic components with frequency or distance from the beam is not as expected from theory. In addition, the observed polarization is not as expected. The amplitude is in general agreement with the predictions of root2.

(18) A conclusion must be that the model does not assume the correct structure of the radiating current. For instance, a current structure as expected from simulations of continuous beam emissions, when modulated by a square wave, will give the observed spectral structure. The amplitude and polarization from such a model may agree better with observations. 


\section{DISCUSSION}

From the 18 summary points given throughout this paper it is seen that a basic understanding of many processes associated with electron beam ejection in space plasmas has been achieved. This knowledge has emerged from a synthesis of experiments, analytical modelling, and computer simulations. As a result, beam ejection can now be controlled to a higher degree than before, allowing the ejection of larger currents, increasing the beam coherence and the escape energy of the beam, and reducing the plasma wave noise. These are crucial skills for some of the applications of electron beams to improving understanding of natural processes of plasma wave generation.

Experiments in the future are needed to study still unresolved issues as well as to capitalize on our ability to better control the electron beam ejection process. High time resolution observations of plasma waves and particles and potential oscillations of beam-emitting platforms are needed to understand the establishment of the payload potential and the sheath structure. Ionization processes in the sheath region due to outgas, thruster emissions or hollow cathode operations also need to be studied. Calculations indicate that instabilities may be induced by the ionization (Cook and Katz, 1988).

One of the natural processes that will need more study in the future regards the generation of electromagnetic radiation from electron beams. While the consensus is that radiation observed in past experiments is a result of Cherenkov radiation, it is still debated how the radiation current structure is formed. It is important to answer this question as it pertains to the generation of VLF waves in the ionosphere from natural processes. The VLF whistler mode is the most dominant wave mode observed in the magnetosphere, and these waves control the energetic electron population (Kennel and Petschek, 1966).

The ideas and experiences of the past will be put to practice in the $A T L A S-1$ space shuttle mission (1992) which will fly the $S E P A C$ electron beam experiment $(7 \mathrm{kV}, 1.6 \mathrm{~mA})$. For the first time in ionospheric beam experiments, a hollow cathode will be used to control the spacecraft potential. Thus the beam should escape with almost its complete and known energy. Optical emissions from the natural aurora as well as from the $S E P A C$ electron beam, as it ionizes the Earth's upper atmosphere, will be observed by the sensitive onboard cameras and spectrographs of the $A E P I$ experiment. The dynamics of the ionization structures created in the upper atmosphere by the beam will be studied by ground-based radars, and attempts will be made to observe VLF radiation on the ground. (The beam can be modulated up to $5 \mathrm{kHz}$.)
The structure of the radiating beam electron current can possibly be deduced from detailed observations of the electromagnetic field in the vicinity of the beam. Such measurements are planned for the CHARGE-2b sounding rocket experiment (1992). The electromagnetic radiation from the beam will be observed from a sub-payload equipped with a sophisticated wave experiment measuring simultaneously three electric and three magnetic field components. The experiment will also allow the study of the limits to vehicle neutralization by thruster emissions.

Finally we point out that the recent development of comparatively small electron linear accelerators now makes possible a whole new class of ionospheric modification experiments (Banks et al., 1991). With electron beams in the megaelectronvolt range it is possible to create substantial ionization from the source altitude down to an altitude of $40 \mathrm{~km}$. In the region from 40 to $60 \mathrm{~km}$, the modification of the fair weather electric potential may result in electric fields of a sufficient magnitude to cause breakdown and discharge in the beam column. The result is the initiation of lightning discharges along the initial beam column and associated VLF wave generation.

Acknowledgements-We appreciate the close collaboration over the years with many individuals, including Profs W. J. Raitt and N. Kawashima, and Drs R. Winglee, P. L. Pritchett, E. G. Reeves, K. J. Harker, B. E. Gilchrist, P. R. Williamson, M. Mandell, I. Katz, N. Myers, S. Sasaki, W. W. L. Taylor, J. Marshall and J. Burch. P.M.B. wishes to express special thanks to Prof. M. Nicolet for his long-term personal encouragement of studies of the dynamics and structure of ionospheric plasmas.

This work was sponsored by NASA under contract NAS835350 and grant NAGW-2350.

\section{REFERENCES}

Abe, Y., Erickson, K. N. and Winckler, J. R. (1988) ELF electric turbulence near an electron beam-emitting rocket in the auroral ionosphere. Planet. Space Sci. 36, 235.

Amme, R. C. (1969) Origin of secondary electrons ejected from gas-covered surfaces by fast neutral beams. $J$. chem. Phys. 50, 1891.

Arnoldy, R. L., Pollock, C. and Winckler, J. R. (1985) The energization of electrons and ions by electron beams injected in the ionosphere. J. geophys. Res. 90, 5197.

Amoldy, R. L. and Winckler, J. R. (1981) The hot plasma environment and floating potentiais of an electron-beamemitting rocket in the ionosphere. J. geophys. Res. 86, 575 .

Banks, P. M., Chappell, C. R. and Nagy, A. F. (1974) A new model for the interaction of auroral electrons with the atmosphere: spectral degradation, backscatter, optical emission, and ionization. J. geophys. Res. 79, 1459.

Banks, P. M., Fraser-Smith, A. C. and Gilchrist, B. E. (1991) Ionospheric modification using relativistic electron beams. AGARD Conference Proceedings, No. 785, pp. 22-1-2217 , Bergen, Norway.

Banks, P. M. and Gilchrist, B. E. (1985) Artificial plasma 
density structures produced by energetic electron beams from rockets and spacecraft. Geophys. Res. Lett. 12, 175.

Banks, P. M., Gilchrist, B. E., Neubert, T., Myers, N., Raitt, W. J., Williamson, P. R., Fraser-Smith, A. C. and Sasaki, S. (1990) CHARGE-2 rocket observations of vehicle charging and charge neutralization. Adv. Space Res. 10, 133.

Banks, P. M. and Nagy, A. F. (1970) Concerning the influence of elastic scattering upon photóelectron transport and escape. J. geophys. Res. 75, 1902.

Banks, P. M. and Raitt, W. J. (1988) Observations of electron beam structure in space experiments. J. geophys. Res. 93, 5811.

Banks, P. M., Raitt, W. J., White, A. B., Bush, R. I. and Williamson, P. R. (1987) Results from the vehicle charging and potential experiment on STS-3. J. Spacecraft Rockets 24, 138.

Beard, D. B. and Johnson, F. S. (1961) Ionospheric limitations on attainable satellite potential. $J$. geophys. Res. 66, 4113.

Bell, T. F. (1968) Artificial production of VLF hiss. $J$. geophys. Res. 73, 4409.

Bernstein, W., Leinbach, H., Kellogg, P. J., Monson, S. J. and Hallinan, T. (1979) Further laboratory measurements of the beam-plasma discharge. J. geophys. Res. 84, 7271.

Bilitza, D. (1986) International reference ionosphere : recent developments. Radio Sci. 21, 343.

Brenning, N. and Mendillo, M. (Editors) (1990) Active experiments/critical ionization velocity. Adv. Space Res. 10, No. 7.

Burke, W. R. (Editor) (1983) Active experiments in space (Alpach Symposium) European Space Agency Scientific and Technical Publications SP-195. Noordwijk, Netherlands.

Bush, R. I., Reeves, G. D., Banks, P. M., Neubert, T., Williamson, P. R., Raitt, W. J. and Gurnett, D. A. (1987) Electromagnetic fields from pulsed electron beam experiments in space: Spacelab-2 results. Geophys. Res. Lett. 14, 1015 .

Cai, D., Neubert, T., Storey, L. R. O., Banks, P. M., Sasaki, S., Abe, K. and Burch, J. L. (1987) ELF oscillations associated with electron beam injections from the space shuttle. J. geophys. Res. 92, 12,451.

Cartwright, D. G. and Kellogg, P. J. (1974) Observations of radiation from an electron beam artificially injected into the ionosphere. J. geophys. Res. 79, 1439.

Cartwright, D. G., Monson, S. J. and Kellogg, P. J. (1978) Heating of the ambient ionosphere by an artificially injected electron beam. J. geophys. Res. 83, 16.

Cooke, D. L. and Katz, I. (1988) Ionization-induced instability in an electron-collecting sheath. $J$. Spacecraft Rockets 25, 132.

Denig, W. F., Maynard, N. C., Burke, W. J. and Maehlum, B. M. (1990) Electric field measurements during supercharging events on the $M A I M I K$ rocket experiment. $J$. geophys. Res. 96, 3601 .

Dietz, L. A. and Sheffield, J. C. (1975) Secondary electron emission induced by 5-30 keV monoatomic ions striking thin oxide films. J. appl. Phys. 46, 4361.

Egeland, A. and Leer, E. (1986) Professor Kr. Birkeland: his life and work. IEEE Trans. Plasma Sci. PS-14, 666.

Erickson, K. N. and Winckler, J. R. (1990) A study of plasma heating, electric fields and plasma flow near an electron beam-emitting rocket system in the polar ionosphere. $J$. geophys. Res. (submitted).

Faelthammer, C.-G. (1986) Magnetosphere-ionosphere interactions-near-earth manifestations of the plasma universe. IEEE Trans. Plasma Sci. PS-14, 616.

Farrell, W. M. (1990) Comments on "Pulsed electron beam emission in space", by Neubert et al., 1988. J. Geomagn. Geoelec. 42, 57.

Farrell, W. M., Gurnett, D. A., Banks, P. M., Bush, R. I. and Raitt, W. J. (1988) An analysis of whistler mode radiation from the Spacelab-2 experiment. J. geophys. Res. 93, 153.

Farrell, W. M., Gurnett, D. A. and Goertz, C. K. (1989) Coherent Cerenkov radiation from the Spacelab 2 electron beam. J. geophys. Res. 94, 443.

Frank, L. A., Paterson, W. R., Ashour-Abdalla, M., Schreiver, D., Kurth, W. S., Gurnett, D. A., Omidi, N., Banks, P. M., Bush, R. I. and Raitt, W. J. (1989) Electron velocity distributions and plasma waves associated with the injection of an electron beam into the ionosphere. $J$. geophys. Res. 94, 6995.

Friedrich, M., Torkar, K. M., Trøim, J. and Mahlum, B. N. (1991) Plasma observations of the active mother-daughter payload Maimik in the lower thermosphere. Planet. Space Sci. 39, 453-468.

Gekelman, W. and Stenzel, R. (1978) Ion sound turbulence in a magnetoplasma. Phys. Fluids 21, 2014.

Gilchrist, B. E. (1991) Ph. D. thesis, Stanford University.

Gilchrist, B. E., Banks, P. M., Neubert, T., Williamson, P. R., Myers, N. B., Raitt, W. J. and Sasaki, S. (1990) Electron collection enhancement arising from neutral gas jets on a charged vehicle in the ionosphere. J. geophys. Res. 95, 2469.

Goerke, R. T., Kellogg, P. J. and Monson, S. J. (1990) An analysis of whistler mode radiation from a $100 \mathrm{~mA}$ electron beam. J. geophys. Res. 95, 4277.

Grandal, B. (Editor) (1982) Artificial particle Beams in Space Plasma Studies (Geilo Symposium). Plenum Press, New York.

Gurnett, D. A., Kurth, W. S., Steinberg, J. T., Banks, P. M., Bush, R. I. and Raitt, W. L. (1986) Whistler-mode radiation from the Spacelab-2 electron beam. Geophys. Res. Lett. 13, 225.

Gurnett, D. A., Shawhan, S. D. and Shaw, R. R. (1983) Auroral hiss, $\mathrm{Z}$ mode radiation, and auroral kilometric radiation in the polar magnetosphere : $D E 1$ observations. J. geophys. Res. 88, 329.

Hallinan, T. J., Winckler, J. R., Malcolm, P., StenbaekNielsen, H. C. and Baldridge, J. (1990) Conjugate echoes of artificially injected electron beams detected optically by means of new image processing. J. geophys. Res. 95, 6519.

Harker, K. J. and Banks, P. M. (1983) Radiation from pulsed electron beams in space plasmas. Radio Sci. 19, 454.

Harker, K. J. and Banks, P. M. (1985) Radiation from long pulse train electron beams in space plasmas. Planet. Space Sci. 33, 953.

Harker, K. J. and Banks, P. M. (1987) Near fields in the vicinity of pulsed electron beams in space. Planet. Space Sci. 35, 11.

Harker, K. J., Neubert, T., Banks, P. M., Fraser-Smith, A. C. and Donohue, D. J. (1991) Ground level signal strength of electromagnetic waves generated by pulsed electron beams in space. Planet. Space. Sci. 39, 1527-1536.

Hastings, D. E. (1987) Enhanced current flow through a plasma cloud by induction of plasma turbulence. $J$. geophys. Res. 92, 7716.

Hayden, H. C. and Utterback, N. G. (1964) Ionization of helium, neon, and nitrogen by helium atoms. Phys. Rev. A 135, 1575. 
Hedin, A. E. (1987) MSIS-86 thermospheric model. J. geophys. Res. 92, 4649.

Hess, W. N., Trichel, M. C., Davis, T. N., Beggs, W. C., Kraft, G. E., Stassinopoulos, E. and Maier, E. J. R. (1971) Artificial aurora experiment: experiment and principal results. J. geophys. Res. 76, 6067.

Holeworth, R. H. and Koons, H. C. (1981) VLF emissions from a modulated electron beam in the auroral ionosphere. J. geophys. Res. 86, 853.

Hwang, Y. S. and Okuda, H. (1989) Low-frequency electrostatic instabilities excited by injections of an electron beam in space. J. geophys. Res. 94, 10103.

Inan, U. S., Pon, M., Banks, P. M., Williamson, P. R., Raitt, W. J. and Shawhan, S. D. (1984) Modulated beam injection from the space shuttle during magnetic conjunctions of STS 3 with the DE 1 satellite. Radio Sci. 19, 487.

Israelson, G. A., and Winckler, J. R. (1979) Effect of a neutral $\mathrm{N}_{2}$ cloud on the electrical charging of an electron beam-emitting rocket in the ionosphere: ECHO IV.J. geophys. Res. 84, 1442.

James, H. G. (1976) VLF saucers. J. geophys. Res. 81, 501.

Junginger, H., Geiger, G., Haerendel, G., Melzner, F., Amata, E. and Higel, B. (1984) A statistical study of dayside magnetospheric electric field fluctuations with periods between 150 and 600 s. J. geophys. Res. 89, 5495.

Kawashima, N. (1982) Experimental studies of the neutralization of a charged vehicle in space and in the laboratory in Japan, in Artificial Particle Beams in Space Plasma Studies (Edited by Grandal, B.), p. 573. Plenum Press, New York.

Katz, I. and Davis, V. A. (1987) On the need for space tests of plasma contactors as electron collectors, paper presented at the 2nd Annual Conference on Tethers in Space, PSN/NASA/ESA, Venice, Italy, 4-8 October.

Kellogg, P. J., Monson, S. J., Bernstein, W. and Whalen, B. A. (1986) Observations of waves generated by electron beams in the ionosphere. J. geophys. Res. 91, 12,065.

Kennel, C. F. and Petschek, H. E. (1966) Limit on stably trapped particle fluxes. J. geophys. Res. 71, 1.

Langley, R. A., Bohdansky, J., Eckstein, W., Mioduszewski, P., Roth, J., Taglauer, E., Thomas, E. W., Verbeek, H. and Wilson, K. L. (1984) Data compendium for plasmasurface interactions. Nucl. Fusion, Special Issue 27, 99.

Langmuir, I. and Blodgett, K. B. (1924) Current limited by space charge flow between concentric spheres. Phys. Rev. $24,49$.

Lavergnat, J. and Lehner, T. (1984) Low frequency radiation characteristics of a modulated electron beam immersed in a magnetized plasma. IEEE Trans. AP-32, 177.

Lavergnat, J., Lehner, T. and Matthieussent, G. (1984) Coherent spontaneous emission from a modulated beam injected in a magnetized plasma. Phys. Fluids 27, 1632.

Liemohn, H. B. (1965) Radiation from electrons in a magnetoplasma. J. Res. natn. Bur. Stand. D 69, 741.

Linson, L. M. (1969) Current-voltage characteristics of an electron emitting satellite in the ionosphere. $J$. geophys. Res. 74, 2368.

Linson, L. M. (1982) Charge neutralization as studied experimentally and theoretically, in Artificial Particle Beams in Space Plasma Studies (Edited by Grandal, B.), p. 573. Plenum Press, New York.

Machlum, B. M., Troim, J., Maynard, N. C., Denig, W. F., Friedrich, M. and Torkar, K. M. (1988) Studies of the electrical charging of the tethered electron accelerator mother-daughter rocket MAIMIK. Geophys. Res. Lett. 15,725 .

Managadze, G. G., Riedler, W., Balebanov, B. M., Friedrich, M. F., Gagua, T. I., Klos, Z., Laliashvili, N. A., Leonov, N. A., Lyakhov, S. B., Martinson, A. A. and Mayorov, A. D. (1983) Plasma processes in the region of electron beam injection from a high-altitude payload, Active Experiments in Space (Alpbach Symposium) (Edited by Burke, W. R.). European Space Agency Scientific and Technical Publications SP-195. Noordwijk, Netherlands.

Managadze, G. G., Riedler, W. K., Friedrich, M. F., Balebanov, V. M., Lalaishvili, A. A., Leonov, N. A., Lyakhov, S. B., Martinson, A. A., Mayorov, A. D., Klos, Z., Zbyszynski, Z. and Torkar, K. M. (1988) Potential observations of an electron emitting rocket payload and other related plasma measurements. Planet. Space Sci. 36, 399.

Mandell, M. J., Lilley, J. R., Jr., Katz, I., Neubert, T. and Myers, N. B. (1990) Computer modelling of current collection by the CHARGE 2 mother payload. Geophys. Res. Lett. 17, 135.

Mansfield, V. N. (1967) Radiation from a charged particle spiralling in a cold magnetoplasma. Astrophys. J. 147, 672 .

Matsumoto, H. and Fukuchi, K. (1985) Computer simulation of particle acceleration and wave excitation by electron beam injection from space shuttle. Geophys. Res. Lett. 12, 61.

McKenzie, J. F. (1967) Radiation losses from a test particle in a plasma. Phys. Fluids 10, 2680.

Melzner, F., Metzner, G. and Antrack, D. (1978) The Geos electron beam experiment $S$ 329. Space Sci. Instrum. 4, 45.

Myers, N. B., Raitt, W. J., Gilchrist, B. E., Banks, P. M., Neubert, T., Williamson, P. R. and Sasaki, S. (1989) A comparison of current-voltage relationships of collectors in the earth's ionosphere with and without electron beam emission. Geophys. Res. Lett. 16, 365.

Myers, N. B., Raitt, W. J., White, A. B., Banks, P. M., Gilchrist, B. E. and Sasaki, S. (1990) Vehicle charging effects during electron beam emission from the $C H A R G E$ 2 experiment. J. Spacecraft Rockets 27, 25.

Neubert, T. and Banks, P. M. (1990) Plasma density enhancements created by the ionization of the Earth's upper atmosphere by artificial electron beams, $A G A R D$ Conference Proceedings, No. 485, pp. 21-1-21-6. Bergen, Norway.

Neubert, T., Bell, T. F. and Storey, L. R. O. (1987) Resonance between coherent whistler mode waves and electrons in the topside ionosphere. J. geophys. Res. 92, 255.

Neubert, T. and Harker, K. J. (1988) Magnetic fields in the vicinity of pulsed electron beams in space. Planet. Space Sci. 36, 469.

Neubert, T., Hawkins, J. G., Reeves, G. D., Banks, P. M., Bush, R. I., Williamson, P. R., Gurnett, D. A. and Raitt, W. J. (1988) Pulsed electron beam emission in space. $J$. Geomagn. Geoelect. 40, 1221.

Neubert, T., Mandell, M. J., Sasaki, S., Gilchrist, B. E., Banks, P. M., Williamson, P. R., Raitt, W. J., Myers, N. B., Oyama, K. I. and Katz, I. (1990a) The sheath structure around a negatively charged rocket payload. J. geophys. Res. 95, 6155.

Neubert, T., Banks, P. M., Gilchrist, B. E., Fraser-Smith, A. C., Williamson, P. R., Raitt, W. J., Myers, N. B. and Sasaki, S. (1990b) The interaction of an artificial electron beam with the earth's upper atmosphere : effects on spacecraft charging and the near-plasmu environment. $J$. geophys. Res. 95, 12,209.

Neubert, T., Harker, K. J., Banks, P. M., Reeves, G. D. 
and Gurnett, D. A. (1990c) Waves generated by pulsed electron beams. Adv. Space Res. 10, 137.

Neubert, T., Sasaki, S., Gilchrist, B. E., Banks, P. M., Williamson, P. R., Fraser-Smith, A. C. and Raitt, W. J. (1991) Observation of plasma wave turbulence generated around large ionospheric spacecraft : effects of motionally induced EMF and of electron beam emission. J. geophys. Res. 96, 9639.

Neubert, T., Taylor, W. W. L., Storey, L. R. O., Kawashima, N., Roberts, W. T., Reasoner, D. L., Banks, P. M., Gurnett, D. A., Williams, R. L. and Burch, J. L. (1986) Waves generated during electron beam emissions from the space shuttle. J. geophys. Res. 91, 11,321.

O'Neil, R. R., Bien, F., Burt, D., Sandock J. A. and Stair, A. T. Jr. (1978a) Summarized results of the artificial auroral experiment, Precede. J. geophys. Res. 83, 3273.

O'Neil, R. R., Shepherd, O., Reidy, W. P., Carpenter, J. W., Davis, T. N., Newell, D., Ulwick, J. C. and Stair, A. T. Jr. (1978b) Excede 2 test, an artificial auroral experiment : ground-based optical measurements. J, geophys. Res. 83, 3281.

Ohnuki, S. and Adachi, S. (1984) Radiation of electromagnetic waves from an electron beam antenna in an jonosphere. Radio Sci. 19, 925.

Okuda, H. and Ashour-Abdalla, M. (1988) Ion-acoustic instabilities excited by the injection of an electron beam in space. J. geophys. Res. 93, 2011.

Okuda, H. and Ashour-Abdalla, M. (1990) Propagation of a nonrelativistic electron beam in three dimensions. $J$. geophys. Res. 9s, 2389.

Okuda, H. and Ashour-Abdalla, M. (1991) Injection of an overdense electron beam in space. J. geophys. Res. 95, $21,307$.

Omura, Y. and Matsumoto, H. (1988) Computer experiments on whistler and plasma wave emissions for the Spacelab-2 electron beam. Geophys. Res. Lett. $15,319$.

Parker, L. W. and Murphy, B. L. (1967) Potential buildup on an electron-emitting satellite in the ionosphere. $J$. geophys. Res. 74, 1631.

Patterson, M. (1987) Hollow cathode-based plasma contactor experiments for electrodynamic tether. $A I A A$, AIAA-87-0571.

Peratt, A. L. (1986) Evolution of the plasma universe: 1. Double radio galaxies, quasars, and extragalactic jets. IEEE Trans. Plasma Sci. PS-14, 639.

Peratt, A. L. (1988) The role of particle beams and electrical currents in the plasma universe. Laser Particle Beams 6, 471.

Peratt, A. L. (1990) The evidence for electrical currents in cosmic plasma. IEEE Trans. Plasma Sci. PS-18, 26-32.

Pritchett, P. L. (1990) Spatial coherence during pulsed injection of electron beams. J. geophys. Res. 95, 10671.

Pritchett, P. L. (1991) A three-dimensional simulation model for electron beam injection experiments in space. $J$. geophys. Res. 96, 13,781.

Pritchett, P. L., Karamabadi, H. and Omidi, N. (1989) Generation mechanism of whistler waves produced by electron beam injection in space. Geophys. Res. Lett. 16, 883.

Pritchett, P. L. and Winglee, R. M. (1987) The plasma environment during particle beam injection into space plasmas, 1. Electron beams. J. geophys. Res. 92, 7673.

Reeves, E. G. D., Banks, P. M., Neubert, T., Bush, R. I., Williamson, P. R., Fraser-Smith, A. C., Gurnett, D. A. and Raitt, W. J. (1988a) VLF wave emissions by pulsed and DC electron beams in space 1 : Spacelab-2 observations. J. geophys. Res. 93, 14,699.
Reeves, G. D., Banks, P. M., Fraser-Smith, A. C., Neubert, T., Bush, R. I., Gurnett, D. A. and Raitt, W. J. (1988b) VLF wave stimulation by puised electron beams injected from the space shuttle. J. geophys. Res. 93, 162.

Reeves, G. D., Banks, P. M., Neubert, T., Harker, K. J. and Gurnett, D. A. (1990a) VLF wave emissions by pulsed and DC electron beams in space 2 : analysis of Spacelab2 results. $J$. geophys. Res. 95,6505 .

Reeves, G. D., Banks, P. M., Neubert, T., Harker, K. J., Gurnett, D. A. and Raitt W. J. (1990b) Spacelab-2 electron beam wave stimulation: studies of important parameters. J. geophys. Res. 95, 10,655.

Reme, H. (Editor) (1980) Special issue on the results of the active French-Soviet Araks experiments. Ann. Geophys. 36, 3.

Sagdeev, R. Z., Managadze, G. G., Mayorov, A. D., Kyakov, S. B., Martinson, A. A., Romanovsky, Yu. A., Adeiskvily, T. G., Leonov, N. A. and Gagua, T. I. (1981) Peculiarities of the environment disturbance during the electron beam injection from the rocket. Adv. Space Res. 1,77 .

Sasaki, S., Kawashima, N., Kuriki, K., Yanagisawa, M., Obayashi, T., Roberts, W. T., Reasoner, D. L., Taylor, W. W. L., Williamson, P. R., Banks, P. M. and Burch, J. L. (1985a) Ignition of beam plasma discharge in the electron beam experiment in space. Geophys. Res. Lett. 12, 647.

Sasaki, S., Kawashima, N., Kuriki, K., Yanagisawa, M., Obayashi, T., Roberts, W. T., Reasoner, D. L., Taylor, W. W. L., Williamson, P. R., Banks, P. M. and Burch, J. L. (1985b) An enhancement of plasma density by neutral gas injection observed in SEPAC Spacelab-1 experiment. J. Geomagn. Geoelec. 37, 883.

Sasaki, S., Oyama, K. I., Kawashima, N., Watanabe, Y., Obayashi, T., Raitt, W. J., White, A. B., Banks, P. M., Williamson, P. R., Sharp, W. F., Yokota, T. and Hirao, K. (1987) Results from a series of tethered rocket experiments. J. Spacecraft Rockets $24,444$.

Sasaki, S., Oyama, K. I., Kawashima, N., Obayashi, T., Hirao, K., Raitt, W. J., Myers, N. B., Williamson, P. R., Banks, P. M. and Sharp, W. F. (1988) Tethered rocket experiment (CHARGE 2): initial results on electrodynamics. Radio $S c i .23,975$.

Shawhan, S. D., Murphy, G. B., Banks, P. M., Williamson, P. R. and Raitt, W. J. (1984) Wave emissions from dc and modulated electron beams on STS-3. Radio Sci. 19, 471.

Stenzel, R. L. (1978a) Experiments on current-driven threedimensional ion sound turbulence. Part 1 : return-current limited electron beam injection. Phys. Fluids 21, 93.

Stenzel, R. L. (1978b) Experiments on current-driven threedimensional ion sound turbulence. Part 2 : wave dynamics. Phys. Fluids $21,99$.

Stix, T. H. (1962) The Theory of Plasma Waves. McGrawHill, New York.

Szapiro, B. and Rocca, J. J. (1989) Electron emission from glow-discharge cathode materials due to neon and argon bombardment. J. appl. Phys. 65, 3713.

Szapiro, B., Rocca, J. J. and Prabhuram, T. (1988) Electron yield of glow discharge cathode material under helium ion bombardment. Appl. Phys. Lett. 53, 358.

Taylor, W. W. L., Roberts, W. T., Reasoner, D. L., Chappell, C. R., Watkins, J. R., Baker, B. B., Burch, J. L., Gibson, W. C., Black, R. K., Tomlinson, W. M., Ferguson, G. A., Bounds, J. R., Womack, W. M., Banks, P. M., Williamson, P. R., Neubert, T., Williamson, W. S., Obayashi, T., Nagamoto, M., Kawashima, N., Kuriki, K., Ninomayo, K., Sasaki, S., Yanagisawa, M., Ejiri, M. and Kudo, 1. (1991) 
Space experiments with particle accelerators. J. Spacecraft Rockets (in press).

Taylor, W. W. L. and Shawhan, S. D. (1974) A test of incoherent Cerenkov radiation for VLF hiss and other magnetospheric emissions. J. geophys. Res. 79, 105.

Utterback, N. G. and Miller, G. H. (1961) Fast molecular nitrogen beam. Rev. Sci. Instrum. 32, 1101.

Waterman, J., Wilhelm, K., Torkar, K. M. and Riedler, W. (1988) Space shuttle charging or beam-plasma discharge: What can electron spectrometer observations contribute to solving the question? J. geophys. Res. 93, 4134.

Wilhelm, K., Stuedmann, W. and Riedler, W. (1984) Electron flux intensity distributions observed in response to particle beam emissions. Science 225, 186.

Williams, J. D., Wilbur, P. J. and Monheiser, J. M. (1987) Experimental validation of a phenomenological model of the plasma contacting process, paper presented at the 2nd Annual Conference on Tethers in Space, PSN/ NASA/ESA, Venice, Italy, October 48.

Winckler, J. R. (1980) The application of artificial electron beams to magnetospheric research. Rev. Geophys. Space Phys. 18, 649.

Winckler, J. R., Arnoldy, R. L. and Hendrickson, R. A. (1975) Echo 2: a study of electron beams injected into the high-latitude ionosphere from a large sounding rocket. $J$. geophys. Res. 80, 2083.

Winckler, J. R., Erickson, K. N., Abe, Y., Steffen, J. E. and Malcolm, P. R. (1985) ELF wave production by an electron beam emitting rocket system and its suppression on auroral field lines : evidence for Alfven and drift waves. Geophys. Res. Lett. 12, 457.

Winckler, J. R., Malcolm, P. R., Arnoldy, R. L., Burke, W. J., Erickson, K. N., Ernstmeyer, J., Franz, R. C., Hallinan, T. J., Kellogg, P. J., Monson, S. J., Lynch, K. A., Murphy, G. and Nemzek, R. J. (1989) Echo 7: an electron beam experiment in the magnetosphere. EOS 70, 657.

Winckler, J. R., Steffen, J. E., Malcolm, P. R. Erickson, K. N., Abe, Y. and Swanson, R. L. (1984) Ion resonances and ELF wave production by an electron beam emitting rocket in the ionosphere : ECHO 6. J. geophys. Res. 89, 7565.

Winglee, R. M. (1990) Electron beam injection during active experiments: 2. Collisional effects. J. geophys. Res. 95, 6191.

Winglec, R. M. and Kellogg, P. J. (1990) Electron beam injection during active Experiments: 1. Electromagnetic wave emissions. J. geophys. Res. 95, 6167.

Winglee, R. M. and Pritchett, P. L. (1988) Comparative study of cross-field and field-aligned electron beams in active experiments. J. geophys. Res. 93, 5823.

Wong, H. K. and Lin, C. S. (1990) Plasma instabilities of a finite-radius beam in a uniform plasma. Radio Sci. 25, 277.

Yeh, K. C. (Editor) Radio Science (1984) Special issue on emissions from particle beams in space 19, 453. (Guest Editor E. R. Schmerling). 